\title{
Microsurgical Anatomy of the Temporal Lobe and Its Implications on Temporal Lobe Epilepsy Surgery
}

\author{
Baris Kucukyuruk, ${ }^{1}$ R. Mark Richardson, ${ }^{2}$ Hung Tzu Wen, ${ }^{3}$ \\ Juan Carlos Fernandez-Miranda, ${ }^{4}$ and Albert L. Rhoton Jr. ${ }^{1}$ \\ ${ }^{1}$ Department of Neurosurgery, University of Florida, Gainesville, FL 32610, USA \\ ${ }^{2}$ Epilepsy Surgery Program, Department of Neurosurgery, University of Pittsburg, Pittsburgh, PA 15213, USA \\ ${ }^{3}$ Division of Neurosurgery, College of Medicine, University of Sao Paulo, 01232-010 Sao Paulo, SP, Brazil \\ ${ }^{4}$ Department of Neurological Surgery, University of Pittsburgh Medical Center, Pittsburgh, PA 15213, USA
}

Correspondence should be addressed to Albert L. Rhoton Jr., rhoton@neurosurgery.ufl.edu

Received 30 November 2011; Revised 6 February 2012; Accepted 7 February 2012

Academic Editor: Seyed M. Mirsattari

Copyright ( $) 2012$ Baris Kucukyuruk et al. This is an open access article distributed under the Creative Commons Attribution License, which permits unrestricted use, distribution, and reproduction in any medium, provided the original work is properly cited.

Objective. We review the neuroanatomical aspects of the temporal lobe related to the temporal lobe epilepsy. The neuronal, the ventricular, and the vascular structures are demonstrated. Methods. The previous articles published from the laboratory of the senior author are reviewed. Results. The temporal lobe has four surfaces. The medial surface has a complicated microanatomy showing close relation to the intraventricular structures, such as the amygdala or the hippocampus. There are many white matter bundles in the temporal lobe showing relation to the extra- and intraventricular structures. The surgical approaches commonly performed to treat temporal lobe epilepsy are discussed under the light of these data. Conclusion. A thorough knowledge of the microanatomy is necessary in cortical, subcortical, and intraventricular structures of the temporal lobe to achieve better results.

\section{Introduction}

This paper describes the anatomic features of the temporal lobe which are important in the most commonly performed surgical approaches for temporal lobe epilepsy (TLE): the anterior temporal lobectomy (ATL), the transcortical selective amygdalohippocampectomy (TCAH), and the transsylvian selective amygdalohippocampectomy (TSAH). The differences between these approaches and the expected outcomes are best understood by knowing their microanatomical differences.

Two main objectives in epilepsy surgery are removal of the epileptogenic tissue and avoidance of surgical morbidity. Three approaches will be reviewed from the perspective of (1) avoidance of visual pathways (optic tract, lateral geniculate body Meyer's loop, and optic radiations), (2) white matter pathways involved in the neurocognitive sequelae, (3) extent of the incision to the temporal stem, (4) extent of amygdalectomy, and (5) avoidance of vascular injury.

\section{Neural Features}

2.1. Cortical Anatomy. The temporal lobe has four surfaces: lateral, medial, superior, and inferior (Figure 1(a)).

2.1.1. Lateral Surface. The lateral cortical surface of the temporal lobe is located below the sylvian fissure. While its anterior and inferior limits are natural bone structures, the temporal lobe is separated posteriorly from the occipital lobe by the lateral parietotemporal line, an imaginary line connecting the preoccipital notch and the parietooccipital sulcus, and it is also separated from the parietal lobe by the occipitotemporal line, a line connecting the most posterior limit of sylvian fissure with the lateral parietotemporal line (Figure 1(a)) [1]. The lateral surface of temporal lobe consists of three gyri: superior, middle, and inferior, which are separated by two parallel sulci: superior and inferior. While the superior temporal sulcus lies between the superior and middle temporal gyri, the inferior temporal sulcus courses 


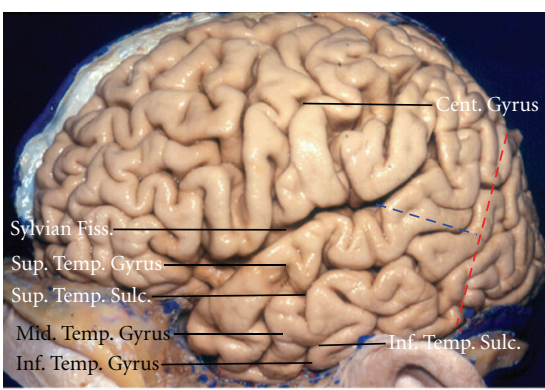

(a)

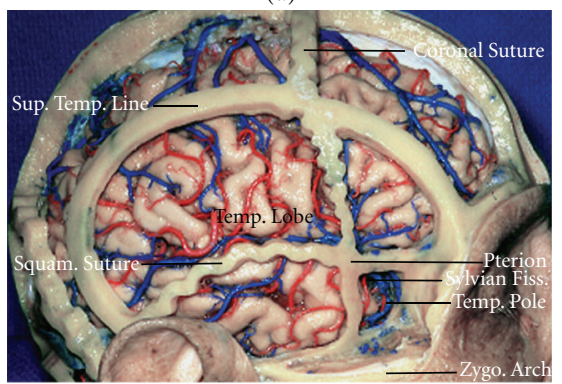

(c)

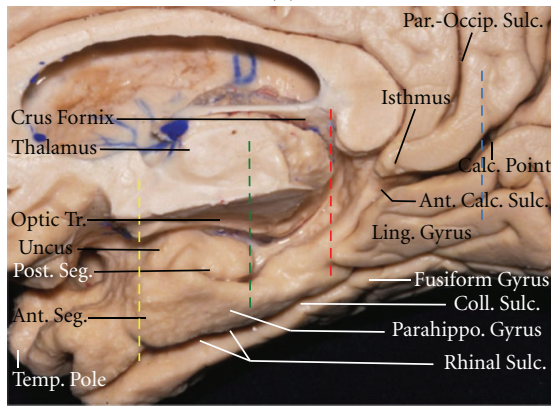

(d)

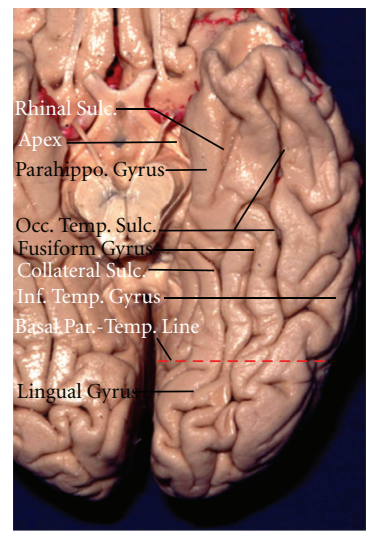

(b)

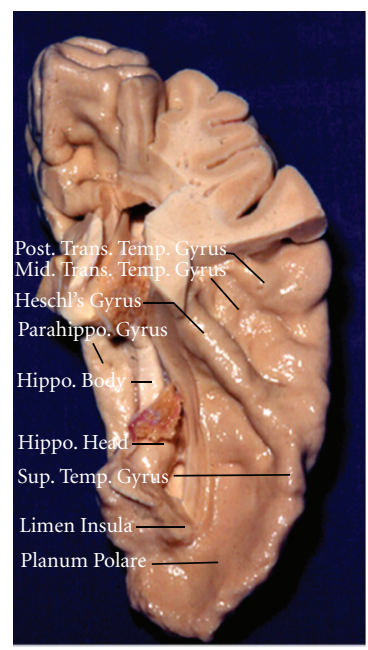

(e)

FIgURE 1: (a) Lateral view of the left hemisphere. The lateral surface of the temporal lobe consists of three parallel gyri: superior, middle, and inferior temporal gyri. These gyri are separated by the superior and inferior temporal sulci. The lateral parietotemporal line (red dashed line), an imaginary line connecting the preoccipital notch and parietooccipital sulcus, separates the temporal and occipital lobes, and the occipitotemporal line (blue dashed line), an imaginary line connecting the posterior margin of the sylvian fissure with lateral parietotemporal line, separates the temporal and parietal lobes. (b) Inferior view of the left temporal lobe. The basal surface of the temporal lobe consists of, from lateral to medial, the inferior margin of the inferior temporal gyrus, the fusiform gyrus, and the parahippocampal gyrus. The fusiform gyrus is separated laterally from the inferior temporal gyrus by the occipitotemporal sulcus, and medially from the parahippocampal gyrus by the collateral posteriorly and rhinal sulci anteriorly, which are not continuous in every case. The basal parietotemporal line connecting the preoccipital notch and inferior end of parietooccipital sulcus separates the temporal and occipital lobes at the basal surface. (c) The relationship of the temporal lobe with bony structures in a right hemisphere. The cranial sutures and the superior temporal line have been preserved, and the dura has been opened. The pterion is located at the lateral margin of the sphenoid ridge near the junction of the coronal, squamosal, and frontosphenoid sutures and the lateral end of the greater sphenoid wing and stem of the sylvian fissure. The squamosal suture follows the anterior part of the posterior limb of the sylvian fissure before turning downward, at the level of the postcentral and supramarginal gyri, to cross the junction of the middle and posterior third of the temporal lobe. The pole of the temporal pole fits into the cupped inner surface of the greater wing of the sphenoid bone. Most of the lateral surface of the temporal lobe is positioned deep to the squamous part of the temporal bone, except, the posterior part of the lateral surface extending deep to the parietal bone. The basal surface of the temporal lobe sits on the floor of the middle fossa and is positioned at the level of the upper edge of the zygomatic arch. (d) The medial view of the temporal lobe in a right hemisphere. This region is divided into three segments: anterior, middle, and posterior. The anterior segment begins at where the rhinal sulcus turns upward at the posterior edge of the temporal pole (yellow interrupted line) to a vertical line crossing the posterior edge of the uncus (green interrupted line), the middle segment extends from this point to the level of the quadrigeminal plate (red interrupted line), and the posterior segment extends from the quadrigeminal plate to the calcarine point (blue interrupted line) located at the junction of the parietooccipital and calcarine sulci. (e) The superior view of the left temporal lobe. This surface facing the the sylvian fissure is divided, from anterior to posterior, in three portion: the planum polare, the anterior transverse temporal gyrus, referred to as the Heschl's gyrus, and the planum temporale containing the middle and posterior transvers temporal gyri. Ant.: anterior; Cent., central; Calc.: calcarine; Fiss.: fissure; Hippo.: hippocampus; Inf.: inferior; Mid.: middle; Occ.: occipital; Parahippo.: parahippocampal; Par.-Occip.: parieto-occipital; Par.-Temp.: parietotemporal; Post.: posterior; Squam.: squamous; Sulc.: sulcus; Sup.: superior; Temp.: temporal; Trans.: transvers Tr.: tract; Zygo.: zygomatic. 
between the middle and the inferior temporal gyri. The superior temporal gyrus is continuous with the transverse temporal gyri on the temporoopercular surface. The angular gyrus, a parietal lobe structure, caps the most posterior end of the superior temporal sulcus. The temporal gyri, especially the inferior temporal gyrus, are often separated into small parts by sulcal bridges (Figure 1(a)). Ono et al. stated that the inferior temporal sulcus is separated into three or more parts in $92 \%$ of cases [2].

2.1.2. Superior Surface. The superior surface of temporal lobe faces the sylvian fissure and the sylvian cistern. This surface, from anterior to posterior, has three parts: the planum polare, the anterior transverse temporal gyrus or Heschl's gyrus, and the planum temporale (Figure 1(e)). The planum polare is a relatively flat surface without any gyri on the anterior part of the superior surface. It is limited posteriorly by the Heschl's gyrus, the most anterior of the transverse temporal gyri, which blends around the margin of the sylvian fissure into the superior temporal gyrus. Posterior to the Heschl's gyrus lies the planum temporale, which consists of middle and posterior transverse temporal gyri [3].

The sylvian fissure is a very important landmark on the lateral surface of the cerebrum. It crosses deep between opercular surfaces of the frontal, parietal, and temporal lobes reaching the carotid cistern anteriorly and the insular surface posteriorly. The superficial part of the sylvian fissure has a stem and three rami. The stem begins at the anterior clinoid process in the frontobasal area, extends lateral and adjacent to the sphenoid ridge, and divides at the surface into three rami: anterior horizontal, anterior ascending, and posterior. The deep part of the Sylvian fissure, referred to as the Sylvian cistern, has two compartments: the sphenoidal and the operculoinsular. The sphenoidal compartment is positioned between the carotid cistern medially, the posterior part of the frontoorbital area superiorly and the planum polare of the temporal lobe, and the amygdala inferiorly. The operculoinsular compartment is formed by two clefts: opercular and insular. The opercular cleft lies between the opercular surfaces of frontal and parietal lobes superiorly, and opercular surface of the temporal inferiorly. The insular cleft has an upper lip that lies between the frontal and the parietal lobes and the insular surface, and a lower lip that lies between the temporal lobe and the insular surface [4].

2.1.3. Inferior Surface. The sulci and gyri, which constitute the inferior surface of the temporal lobe, are continuous with the basal surface of the occipital lobe (Figure 1(b)). This surface is formed by three gyri and two longer and one shorter sulci $[2,5]$. Of these longer sulci, the occipitotemporal sulcus courses laterally and separates the lower surface of the inferior temporal gyrus and the fusiform gyrus. The other longer sulcus, the collateral sulcus, separates the fusiform gyrus from the medially placed parahippocampal gyrus. The rhinal sulcus, the shorter sulcus, courses at the lateral edge of the uncus between the uncus and the fusiform gyrus and may or may not be continuous with the collateral sulcus. The indentation of the collateral sulcus towards the temporal horn generates a prominence at the floor of the temporal horn, called the collateral eminence, and at the floor of the atrium, called the collateral trigone (Figures 3(f), 5(a), 5(e), and $5(\mathrm{f}))$.

2.1.4. Medial Surface. The medial surface of the temporal lobe, or medial temporal region (MTR), is the most complicated of cortical surfaces. For a better understanding, Fernández-Miranda et al. divided this surface into three segments: anterior, middle, and posterior [6]. The anterior segment begins where the rhinal sulcus turns superiorly at the anterior edge of the uncus and ends at the posterior limit of the uncus; the medial segment begins at the posterior edge of the uncus and ends at the level of the quadrigeminal plate; the posterior segment begins at this point and ends at the calcarine point where the parietooccipital and calcarine sulci join (Figure 1(d)).

The anterior MTR is formed mostly by the uncus and the entorhinal cortex. The uncus has an anterior and a posterior segments, which come together at a medially directed prominence, the apex of the uncus. The anterior segment of the uncus belongs to the parahippocampal gyrus and contains two gyri: the semilunar gyrus and the ambient gyrus. The semilunar gyrus is positioned on the upper part of the anterior segment, above the cortical nucleus of amygdala. The semilunar gyrus is separated from the anterior perforated substance by the entorhinal sulcus and optic tract superolaterally and from the ambient gyrus by the semiannular sulcus medially and anteriorly. The ambient gyrus, formed mainly by the entorhinal cortex, occupies the anterior and inferior parts of this segment (Figures $2(a)-2(c))$. The posterior segment of uncus has two parts, superior and inferior, which are separated by the uncal sulcus. The inferior part, formed by the parahippocampal gyrus, is occupied by the entorhinal area. The entorhinal area also occupies the inferior surface of the anterior segment of the uncus and is limited on the lateral side by the rhinal sulcus anteriorly and the collateral sulcus posteriorly. The posterior limit of the entorhinal area is accepted as the posterior limit of the uncus. The entorhinal area has an important role in afferent and efferent connections of the hippocampus [1]. The superior part of the uncus is formed by the hippocampal head and has the fimbria of fornix at its posterior limit. This part is the site of three small gyri, the uncinate gyrus, the band of Giacomini, and the intralimbic gyrus. The band of Giacomini is the continuation of the dentate gyrus. The intralimbic gyrus contains the CA3 and the CA4 sectors of the hippocampal formation. The anterior segment of the uncus faces the carotid cistern, the internal carotid artery (ICA), and the proximal M1 segment of middle cerebral artery (MCA); the apex of the uncus faces the oculomotor nerve; the posterior segment of the uncus faces the crural cistern, the posterior cerebral artery (PCA) below and the anterior choroidal artery (AChA) above, and the crus cerebri.

The middle MTR, when viewed medially, is formed from inferior to superior by the parahippocampal gyrus, the dentate gyrus, and the fimbria of fornix (Figure 2(a)). The fimbriodentate sulcus separates the fimbria of fornix 


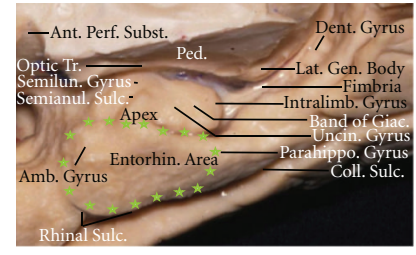

(a)

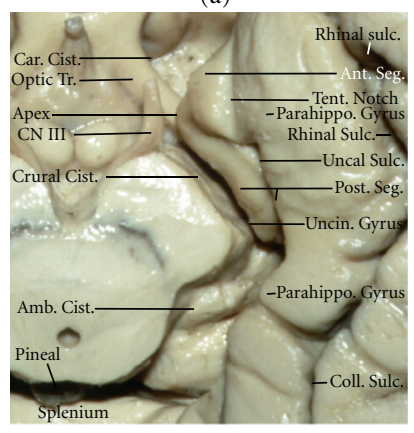

(c)

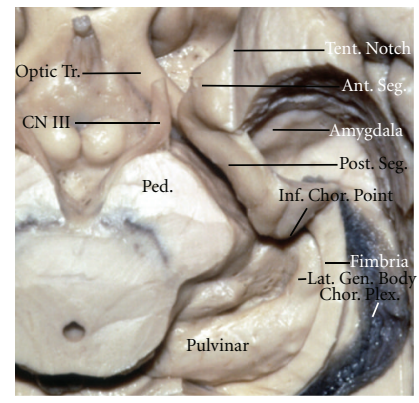

(e)

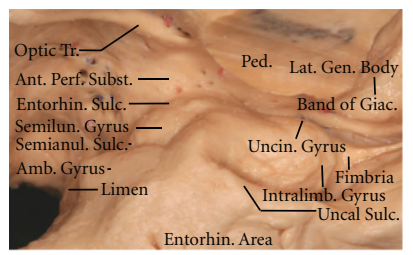

(b)

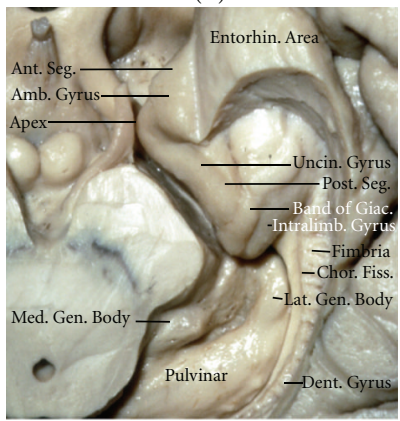

(d)

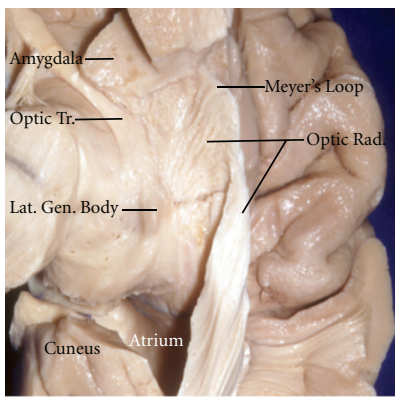

(f)

FIGURE 2: (a) Enlarged view of the anterior and middle segments of the medial temporal region (MTR). The anterior segment is formed by the uncus and the entorhinal area. The uncus is divided into anterior and posterior segments meeting at the medially directed apex. The entorhinal area shown with green stars occupies the inferior surfaces of the anterior and posterior segments of the uncus but does not have a clearly demarcated borders. The parahippocampal gyrus forming the cortical component of the middle segment of the MTR has 3 surfaces: inferior, medial, and superior. The superior surface faces the lower surface of the pulvinar across the upper part of the ambient cistern and has superiorly the dentate gyrus and the fimbria of the fornix. (b) Enlarged view of the anterior segment of the MTR. The anterior segment of the uncus contains the semilunar gyrus and the ambient gyrus. The semilunar gyrus covers the cortical nucleus of the amygdala. The ambient gyrus located anterior and inferior to the semilunar gyrus is separated by the semiannular sulcus from it. The posterior segment of the uncus is divided into an upper and lower part by the uncal sulcus. The upper part is formed by the medially folded extraventricular (cisternal) head of the hippocampus, and the lower part is formed by the anterior part of the parahippocampal gyrus. The intralimbic gyrus forms theposterior end of the uncus and is the site of attachment of the fimbria. (c-f) Stepwise dissection of the basal surface of the temporal lobe. (c) The anterior segment of the uncus faces the carotid cistern, and posterior segment faces the crural cistern and the cerebral peduncle. The uncal apex is positioned lateral to the oculomotor nerve. The cortical component of the middle MTR formed by the parahippocampal gyrus faces the midbrain across the ambient cistern. (d) The part of the posterior uncal segment located below the uncal notch has been removed to expose the lower surface of the upper part of the posterior uncal segment formed by the extraventricular head of the hippocampus. The fimbria is situated above the dentate gyrus. The choroidal fissure, located between the thalamus and fimbria, extends along the lateral edge of the lateral geniculate body and pulvinar. (e) The hippocampus and dentate gyrus have been removed while preserving the fimbria and the choroid plexus attached along the choroidal fissure. The amygdala forms the anterior wall of the temporal horn and fills most of the anterior segment of the uncus. The inferior choroidal point, located at the lower end of the attachment of the choroid plexus in the temporal horn, is positioned behind the head of the hippocampus, anterior to the lateral geniculate body, and lateral to the posterior edge of the cerebral peduncle. (f) The fimbria and choroid plexus have been removed to expose the roof of the temporal horn. The tapetum fibers forming the roof of the temporal horn have been removed to expose the optic radiations arising from the lateral geniculate body and passing across the roof and around the lateral wall of the temporal horn. Amb.: ambient; Ant.: anterior; Car.: carotis; Chor.: choroidal; Cist.: cistern; CNIII.: oculomotor nerve; Coll.: collateral; Dent.: dentate; Entorhin.: entorhinal; Fiss.: fissure; Gen.: geniculate; Giac.: Giacomini; Inf.: inferior; Intralim.: intralimbic; Lat.: lateral; Parahippo.: parahippocampal; Ped.: peduncle; Perf.: perforated; Plex.: plexus; Post.: posterior; Rad.: radiation; Seg.: segment; Semianul.: semianuler; Semilun.: semilunar; Subst.: substantia; Sulc.: sulcus; Tent.: tentorial; Tr.: tract; Uncin.: uncinate. 


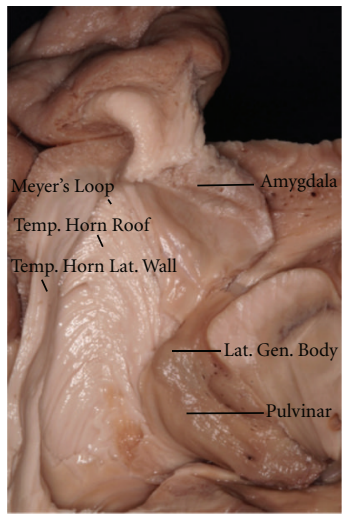

(a)

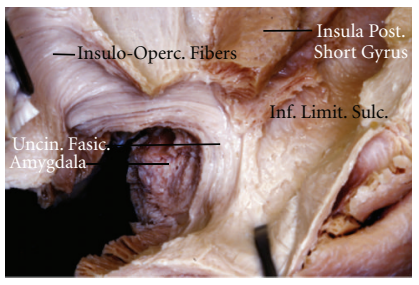

(d)

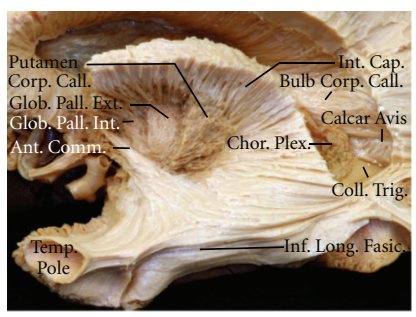

(f)

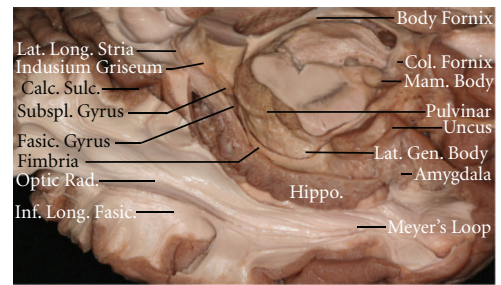

(b)

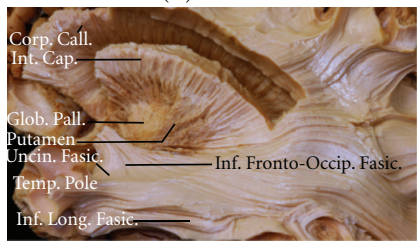

(c)

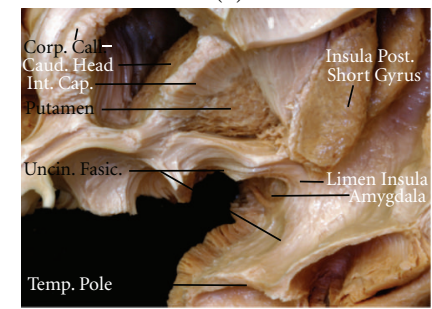

(e)

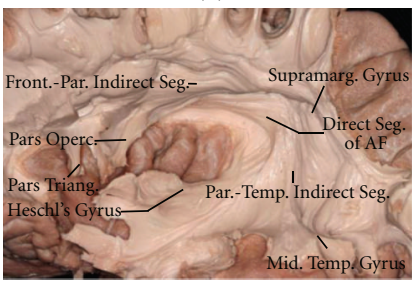

(g)

FIGURE 3: (a) The inferior view of the roof of the temporal horn. The roof and the lateral wall of the temporal horn are formed by the tapetum fibers of the corpus callosum, which is covered by optic radiations with the only exception of the anterior portion of the lateral wall. Optic radiations arise from lateral geniculate body as three unseperable bundles. The anterior bundle making a prominent anterior curve is referred to as the Meyer's loop. (b) Inferomedial view of the left hemisphere. The cortical gray matter of the isthmus and the lingual, parahippocampal, and fusiform gyri have been removed. The cingulum travels inside the isthmus and parahippocampal gyrus. The fasciolar gyrus and its continuation, the subsplenial gyrus, form part of the hippocampal tail below the splenium. (c) Superolateral view of the temporal subcortical structures, the central core, and the lateral ventricular space. The insula and the underlying extreme and external capsules have been removed to expose the central core. The internal capsule course between the caudat nucleus medially and lentiform nucleus laterally. The corpus callosum forms the anterior wall and the roof of the lateral ventricle. The uncinate fasciculus (UF) and the inferior occipitofrontal fasciculus (IFOF) form the temporal stem. The UF forming the anterior part of the temporal stem interconnects the orbitofrontal cortex and the anterior part of the temporal lobe. (d) Closer view of the UF. The UF travels in the extreme and extrenal capsules and deep to the limen insula to connect the the orbitofrontal cortex to the temporal pole and the amygdala. (e) The view of the left hemisphere. The anterior and middle short gyri of the insula and the callosal fibers forming the anterior portion of the roof of the lateral ventricle have been removed to expose the central core. The lateral wall of the frontal horn is formed by the head of the caudate nucleus situated medial to the internal capsule. The lentiform nucleus formed by the putamen and the globus pallidus is located lateral to the internal capsule. (f) The fibers contributing to the lateral wall of the lateral ventricle and the atrium have been removed. The medial wall of the atrium is built by two prominences. The superior prominence, referred to as the bulp of corpus callosum, is formed by the fibers in the forceps major, and the inferior prominence, referred to as the calcar avis, is the bulge of the calcarine sulcus to the ventricular space. Globus pallidus has an external and internal parts seperated by the internal medullary lamina. The anterior commissure is located ventral to the globus pallidus. The inferior longitudinal fasciculus (ILF) connecting the anterior temporal lobe to the posterior part of the temporal lobe and occipital lobe is situated lateral to the optic radiations at the lateral wall of the temporal horn. (g) The view of the superior longitudinal fasciculus (SLF) in a left hemisphere. The SLF consists of three segments: a frontoparietal segment, a parietotemporal segment, and a frontotemporal segment, referred to as the Arcuate fasciculus providing a direct connection between the speech areas located at the inferior frontal gyrus and the posterior part of the superior temporal gyrus. AF: Arcuate Fasciculus; Ant.: anterior; Calc.: calcarine; Call.: callosum; Cap.: capsule; Chor.: choroidal; Col.: column; Coll.: collateral; Comm.: commissure; Corp. Corpus; Ext.: external; Fasic.: fasciculus; Front.-Occip.: fronto-occipital; Front.-Par.: frontoparietal; Gen.: geniculate; Glob.: globus; Hippo.: hippocampus; Inf.: inferior; Insulo-operc.: insulo-opercular; Int.: internal; Lat.: lateral; Long.: longitudinal; Mam.: mamillary; Mid.: middle; Operc.: operculum; Pall.: pallidus; Post.: posterior; Rad.: radiation; Seg.: segment; Subspl.: subsplenial; Sulc.: sulcus; Supramarg.: supramarginal; Temp.: temporal; Triang.: trianguler; Uncin.: uncinate. 
and the dentate gyrus, and the hippocampal sulcus separates the dentate gyrus and the parahippocampal gyrus. In this region, the subiculum is positioned on the superior surface of the parahippocampal gyrus. The presubiculum, a six-layered modified cortex between the subiculum and the cortex of the parahippocampal gyrus, occupies the medial surface of the parahippocampal gyrus.

The posterior MTR is formed by the posterior end of the parahippocampal gyrus, which is divided by the anterior end of the calcarine sulcus into the isthmus of the cingulate gyrus superiorly and the lingual gyrus inferiorly. Functionally, the parahippocampal gyrus shows a closer functional relationship with the isthmus of the cingulate gyrus, because the cingulum bundle passes from the isthmus into the parahippocampal gyrus. Superiorly, the fimbria of the fornix courses posteriorly to become the crus of the fornix, which wraps around the posterior aspect of the pulvinar. The hippocampal tail passes posterior to blend into the fasciolar gyrus, just below the splenium of the corpus callosum (Figure 3(b)). The quadrigeminal cistern is located medial to the posterior MTR.

2.2. Subcortical Anatomy. The main debate in selecting the best surgical approach for temporal epilepsy, other than the long-term seizure control, focuses on two topics: damage to the optic radiations and memory deficit after the surgery. However, there are clinical series that favor different types of surgery understanding the microanatomy of white matter bundles is important to achieving better results [7-11].

The optic radiations, the geniculocalcarine pathway, have a complex anatomy [12-14]. The visual input is carried to the lateral geniculate body (LGB) of the thalamus via the optic nerve, the optic chiasm, and the optic tract. The LGB is located on the inferolateral side of the thalamus and just posterior to the cisternal side of the inferior choroidal point. The fibers divide into three bundles after leaving the LGB. The posterior bundle passes posteriorly to reach the superior lip of the calcarine sulcus without making any anterior curve. The middle bundle makes a partial anterior curve on its course to the calcarine cortex, and the anterior bundle of optic radiations makes a prominent anterior curve, referred to as the Meyer's loop, at the roof of the temporal horn on its way to the inferior lip of the calcarine sulcus. Damage to the Meyer's loop causes an upper contralateral quadrantanopia. The optic radiations are separated from the temporal horn by a thin layer of tapetal fibers originating from the corpus callosum. The optic radiations completely cover the roof of the temporal horn and exceed the anterior wall of the temporal horn by a few millimeters. They also cover the lateral wall of the temporal horn except its anterior part [1214]. At the level of the atrium, optic radiations cover only the lateral wall; the medial wall of the atrium is always free from the optic radiations [15].

The uncinate fasciculus (UF) and inferior longitudinal fasciculus (ILF) also have important role in epilepsy surgery because of their importance in memory function. The UF connects the anterior temporal lobe with the orbitofrontal cortex by forming a curve deep to the limen insula and courses within the ventral extreme and external capsules. The
UF occupies the anterior part of the temporal stem (Figures $3(\mathrm{c})-3(\mathrm{e}))$ [16]. Its functions are not fully understood [17], but the orbitofrontal cortex and the anterior temporal lobe are reported to have an important role in recognizing faces, actions, and objects and also emotion [18-20].

The ILF courses adjacent to the inferior part of the lateral wall of the temporal horn and is located lateral and inferior to the optic radiations (Figures 3(c) and 3(f)) [21]. The ILF connects the anterior temporal lobe to the fusiform gyrus and dorsolateral parts of the occipital lobe. It is suggested that the ILF has a role in learning and remembering visual stimuli. Cohen et al. stated that the process of learning to read occurs by remembering visual stimuli of words. They suggested that the posterior part of the occipitotemporal sulcus referred to as the "visual word form area" is involved in this process [22]. Therefore, the ILF can be important in learning to read. It was debated, until recently, whether the ILF consists of only long horizontal fibers; however, Catani et al. have shown in their DTI study that the ILF consists of both long horizontal fibers (direct part) and interconnecting $\mathrm{U}$ fibers (indirect part) [23].

The arcuate fasciculus (AF) is considered to be a subsegment of the superior longitudinal fasciculus $[24,25]$. The structure of this white matter bundle was further detailed in a DTI study by Catani et al. [26]. In their study, they proposed that the AF consists of two indirect parts and one direct part. The first indirect part connects the inferior frontal gyrus to the supramarginal gyrus, and the second indirect part connects the supramarginal gyrus to the posterior part of superior temporal gyrus. The direct part of the AF is proposed to connect the inferior frontal gyrus to the posterior temporal areas by coursing dorsal to the insula by forming an arch (Figure $3(\mathrm{~g})$ ). The AF has a major role in language processing, which is thought to involve both a ventral pathway and a dorsal pathway [27]. It is suggested that the AF forms the dorsal pathway and plays a role in the phonological part of language [28]. This subcortical structure functions in the mapping of sound into articulation [29]. Therefore, damage to the AF causes a deficit in the production of speech or appropriate words.

The inferior occipitofrontal fasciculus (IOFF) plays another crucial role in language processing. The IOFF connects the inferior frontal cortex and the dorsolateral prefrontal cortex to the posterior part of the inferior surface of the temporal lobe and to the parts of the occipital lobe superior to the calcarine sulcus. From a functional point, the ventral pathway connecting areas known to have a role in picture naming and object recognition has a semantic role in the naming of sounds or recognition of speech $[30,31]$. In a previous report from the laboratory of the senior author, it was shown that the IOFF occupies the ventral part of the extreme and external capsules and the ventral claustrum (Figure 3(c)) [16].

The IOFF is situated at the posterior two-thirds of the temporal stem. White matter tracts connecting the temporal lobe with other parts of the brain, such as the insula, basal ganglia, and frontal and parietal lobes, course through temporal stem. In the transsylvian route to the MTR, the IOFF may be damaged with the incision to the inferior 
limiting sulcus. Ebeling and von Cramon [32] stated that the temporal stem is the area between the roof of the temporal horn and the inferior limiting sulcus. On the other hand, Choi et al. described the temporal stem as the area between the inferior limiting sulcus, limen insula, entorhinal sulcus, and tail of caudate nucleus [33]. According to this organization, the following fiber tracts are included in the temporal stem: the extreme capsule, the UF, IOFF, the anterior commissure, the ansa peduncularis, and the inferior thalamic peduncle, which includes optic radiations. Separation of these white matter tracts, located around posterior limit of the inferior limiting sulcus, is very difficult. This intermixed structure of fiber tracts, including the IOFF, optic radiations, the anterior commissure, and the ILF, is referred to as the sagittal stratum.

2.3. Ventricular Anatomy. The anatomy of the temporal horn and atrium will be discussed with their relationship to the three parts of the MTR $[6,34]$.

The anterior part of the temporal horn is located lateral to the anterior MTR. The posterior limit of this part is the inferior choroidal point, which is the entry point of the AChA into the temporal horn in most cases (Figures 4(a), $4(\mathrm{f}), 5(\mathrm{f})$, and $5(\mathrm{~g}))[6]$. The inferior choroidal point is located at the lower end of the attachment of the choroid plexus and is positioned just behind the head of the hippocampus. The uncal recess is situated anterior to the hippocampal head and separates it from the amygdala. The uncal recess is the intraventricular counterpart of the uncal apex.

The amygdala sits in the lateral part of the anterior segment of the uncus. It forms the anterior wall and the anterior part of the roof of the temporal horn. The bulge of the amygdala to the ventricle is the intraventricular representation of the semilunar gyrus, which occupies the anterior segment of the uncus. Note that the amygdala in this region is the temporal amygdala and contains the basolateral, the corticomedial, and the central nuclei groups [35]. The extended amygdala is in close relation with the ventral striatum and the anterior commissure and should be avoided during surgery for temporal lobe epilepsy [35]. The amygdala joins with the globus pallidus superomedially without any clear border.

The collateral eminence located lateral to the head of the hippocampus occupies the floor of the temporal horn and is the indentation of the collateral sulcus into the temporal horn (Figures 4(a), 4(d), and 4(f)). The collateral sulcus courses between the parahippocampal gyrus and the fusiform gyrus.

The posterior part of the temporal horn is related to the middle MTR and begins at the inferior choroidal point to open to the atrium. The floor of this part is formed by the collateral eminence similar to the anterior part. The collateral eminence is a reliable landmark to determine the medial limit of the neocortical removal. The medial wall of this part is formed by the body of the hippocampus.

The medial surface of the temporal lobe and the perimesencephalic cisterns can be reached by opening the choroidal fissure. The choroidal fissure begins at the inferior choroidal point, which is located at the posterior limit of the uncus. The choroidal fissure is a natural cleft between the thalamus superiorly and the fimbria of the fornix inferiorly. The choroid plexus is attached on each side to the thalamus and the fimbria of the fornix, via the taenia choroidea and taenia fimbria, respectively. The choroid plexus is adjacent to the body of the hippocampus in this part of the temporal horn. The head of the hippocampus is located in the anterior MTR; therefore, it is not related to the choroidal fissure and the choroid plexus. The ambient cistern can be reached by opening the choroidal fissure in the medial MTR. The roof and the lateral wall of the temporal horn are formed by a layer of tapetal fibers and then are covered by optic radiations. Optic radiations do not cover the most anterior part of the lateral wall of the temporal horn.

The atrium is the ventricular space situated lateral to the posterior MTR. The floor of the atrium is formed by the collateral trigone that is the indentation formed by the posterior end of the collateral sulcus. The intraventricular part of the tail of the hippocampus is located at the most anterior part of the floor. The anterior wall of the atrium has two parts: a lateral part formed by the pulvinar and a medial part formed by the crus of fornix. The medial wall of the atrium is formed by two prominences: the inferior prominence, referred to as the calcar avis, is the indentation of the calcarine sulcus into the ventricular space, and the superior prominence, referred to as the bulb of corpus callosum, is formed by the callosal fibers of the forceps major. The lateral wall of the atrium is formed by the optic radiations.

\section{Vascular Features}

3.1. Arterial Supply. The temporal lobe is close to important vascular structures both arterial and venous. The PCA, the MCA, the AChA, and also the ICA are met in surgical interventions directed to the different parts of the temporal lobe. A clear understanding of microvascular anatomy of the temporal lobe is needed to perform epilepsy surgery with minimal morbidity.

3.1.1. Posterior Cerebral Artery. The PCA has the main role of supplying the MTR and the inferior surface of the temporal lobe. The PCA is divided into 4 segments: P1, P2, P3, and P4 (Figures 4(e) and 4(f)) [36]. The P1 arises at the bifurcation of the basilar artery and is situated medial to the anterior segment of the uncus during its course in the interpeduncular cistern. The P1 ends after posterior communicating artery (PCoA) joining the PCA and the P2 begins. The P2 terminates at the posterior margin of the midbrain and is divided into an anterior segment (P2a) and a posterior segment (P2p). The P2a courses in the crural cistern located between the posterior segment of the uncus and the cerebral peduncle. The P2p courses in the ambient cistern located between the parahippocampal gyrus and the midbrain. The $\mathrm{P} 3$ begins at the posterior part of the ambient cistern, posterolateral to the midbrain, and courses back into the quadrigeminal cistern to end at the anterior end of the 


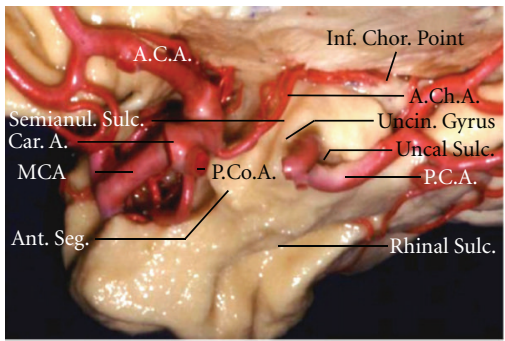

(a)

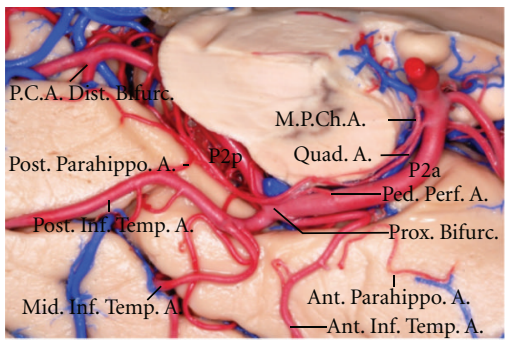

(c)

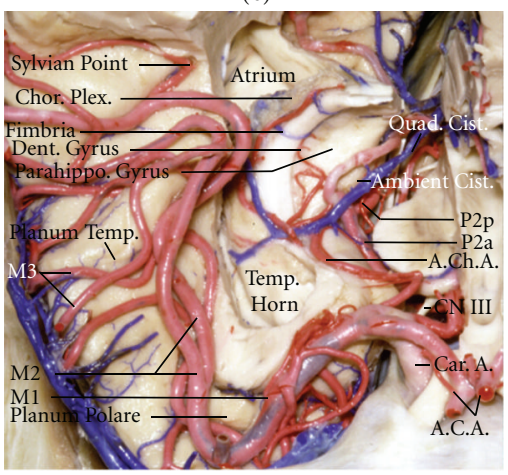

(e)

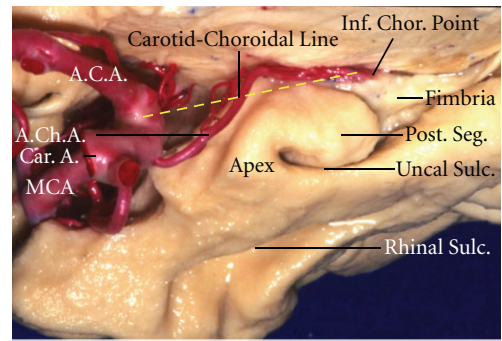

(b)

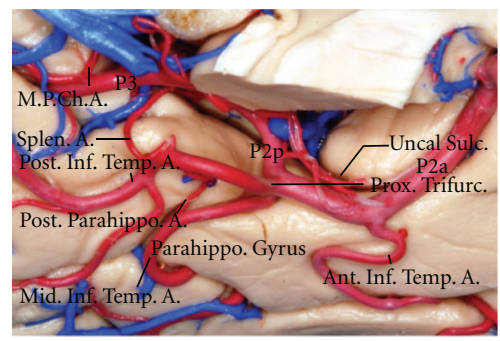

(d)

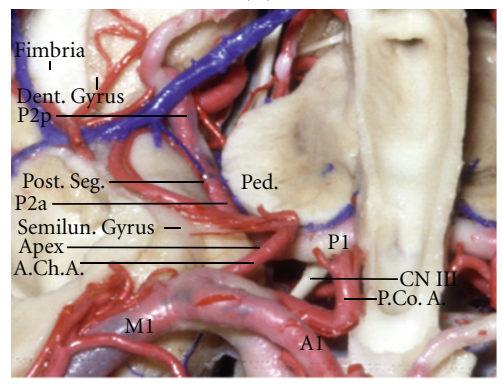

(f)

Figure 4: (a) Medial view of the anterior segment of the medial temporal region (MTR) and adjacent vascular structures. The anterior choroidal artery (AChA) passes above the semiannular sulcus and uncinate gyrus to reach the lower end of the choroidal fissure, where it enters the temporal horn through the inferior choroidal point. The posterior cerebral artery (PCA) courses medial to the uncal sulcus that divides the posterior segment of the uncus into upper and lower parts. (b) The PCA has been removed. An imaginary line (yellow dashed line), referred to as the carotid-choroidal line, drawn from the bifurcation of the internal carotid artery or the M1 segment of the middle cerebral artery (MCA) to the inferior choroidal point is a reliable landmark to determine the superior extent of the amygdalar resection. (c) The PCA is the main arterial supplier of the MTR and typically has a proximal bifurcation situated adjacent to the middle segment of the MTR and a distal bifurcation formed by the posterior inferior temporal artery and the parietooccipital arterial trunk (P2p). The PCA gives off the anterior inferior temporal artery, from which the anterior parahippocampal artery arises, just before the proximal bifurcation. In this case, the middle inferior temporal artery and the posterior parahippocampal artery arise from the posterior inferior temporal artery. The medial posterior choroidal artery and the quadrigeminal artery arise from the proximal part of P2a. (d) The branching pattern of the PCA in another case. A trifurcation into the middle and posterior inferior temporal arteries and the parietooccipital trunk (P2p) was seen instead of the distal bifurcation. The posterior parahippocampal artery arises from the middle inferior temporal artery and the medial posterior choroidal artery from the parietooccipital trunk (P3). (e) The frontal lobe and the central core of the right cerebral hemisphere have been removed to expose the temporal horn, atrium, and the basal cisterns. The M1 segment of the MCA courses on the upper surface of the temporal pole, the M2 segment crosses the insular surface, and the M3 travels on the opercular surfaces. The PCA passes posteriorly in the crural and ambient cisterns ( $\mathrm{P} 2 \mathrm{a}$ and $\mathrm{P} 2 \mathrm{p}$ segments, resp.) to reach the quadrigeminal cistern (P3 segment). (f) Enlarged view of (e). The AChA enters the choroidal fissure at the inferior choroidal point located at the posterosuperior edge of the uncus. A.: artery; A1.: A1 segment of ACA; A.C.A.: anterior cerebral artery; A.Ch.A.: anterior choroidal artery; Ant.: anterior; Bifurc.: bifurcation; Car.: carotid; Ch.: choroidal; Chor.: choroidal; CNIII.: oculomotor nerve; Cist.: cisternal; Dent.: dentate; Dist.: distal; Inf.: inferior; M.C.A.: middle cerebral artery; M1.: M1 segment of MCA; M2.: M2 segment of MCA; M3.: M3 segment of MCA; Mid.: middle; Parahippo.: parahippocampal; P.C.A.: posterior cerebral artery; M.P.Ch.A.: medial posterior choroidal artery; P1.: P1 segment of PCA; P2a.: anterior part of the P2 segment of PCA; P2p.: posterior part of the P2 segment of PCA; P.Co.A.: posterior communicating artery; Ped.: peduncle; Post.: posterior; Quad.: quadrigeminal; Seg.: segment; Semianul.: semiannular; Semilun.: semilunar; Splen.: splenial; Sulc.: sulcus; Temp.: temporal; Trifurc.: trifurcation; Uncin.: uncinate. 


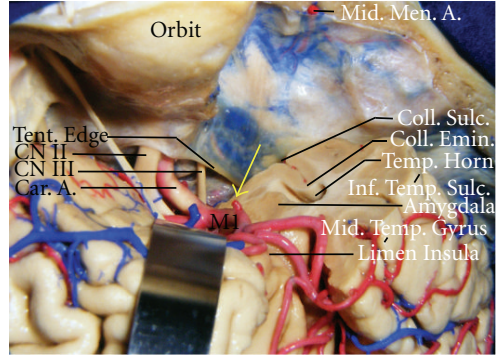

(a)

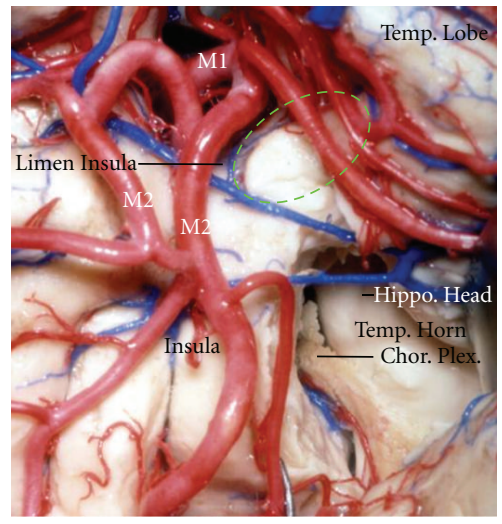

(c)

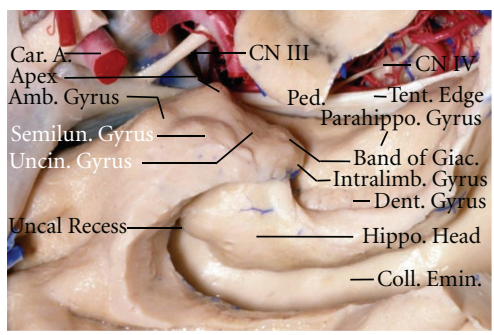

(f)

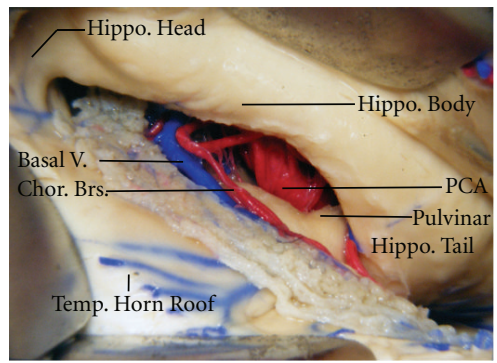

(b)

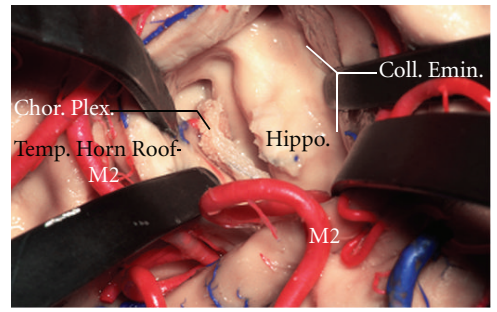

(d)

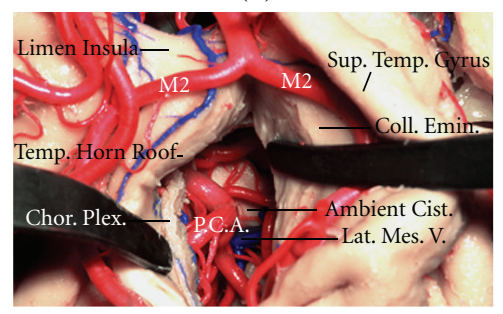

(e)

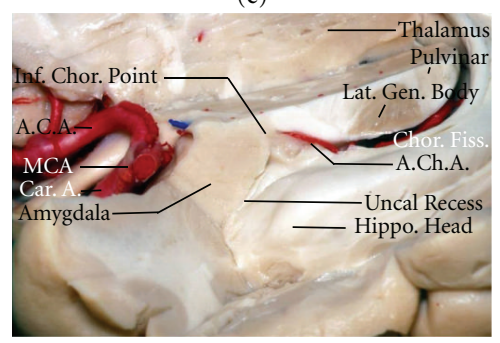

(g)

FIGURE 5: (a) A coronal cut is performed to the specimen positioned as in a pterional craniotomy at the level of limen insula to have a similar view of the anterior temporal lobectomy after the removal of lateral temporal neocortex. The yellow arrow shows the origin of a early cortical branch of MCA. (b) The choroidal plexus is attached to the choroidal fissure with taeniaes to forniceal and thalamic sides. The choroidal fissure should be opened always at forniceal side to prevent both to thalamus and choroidal arteries and veins passing close to thalamic side of the choroid plexus. In this view, arachnoid membrane of the ambient cistern is removed to show the PCA and the basal vein. (c-e) Stepwise dissection of the sylvian fissure, the temporal horn, and the choroidal fissure. (c) The presentation of the amygdala at the temporo-opercular surface is shown in green oval line. An incision in the inferior limiting sulcus has been completed to expose the head of the hippocampus, the choroidal fissure, and the anterior choroidal artery. (d) Closer view of the (c). The roof of the temporal horn has been elevated to have a better view of the temporal horn, the hippocampus, the choroid plexus, and the collateral eminence. (e) Amygdalohippocampectomy has been competed, exposing the vascular elements in the ambient cistern. Inferior surfaces of the anterior and posterior segments of the uncus are related to internal carotid artery and the posterior cerebral artery, respectively. The anterior choroidal artery is related to the superior surfaces of both uncal segments in most cases. (f) Superior view of the left MTR showing the relation of the ventricular and the cisternal components of the MTR to the tentorial edge and cerebral peduncle. The posterior part of the uncus faces the cerebral peduncle across the crural cistern, the apex of the uncus is positioned lateral to the oculomotor nerve. The tentorial edge crosses below the medial part of the uncus. (g) Lateral view of the ventricular surface of the medial temporal lobe. The lateral geniculate body is located just above the choroidal fissure and the middle segment of the medial temporal lobe. The choroidal fissure, along which the choroid plexus (removed) attaches, is located between the fimbria and the thalamus. A.: artery; A.C.A.: anterior cerebral artery; A.Ch.A.: anterior choroidal artery; Amb.: ambient; Ant.: anterior; Brs.: branches; Car.: carotid; Chor.: choroidal; Cist.: cisternal; CNII.: optic nerve; CNIII.: oculomotor nerve; CNIV.: trochlear nerve; Coll.: collateral; Emin.: eminence; Fiss.: fissure; Giac.: Giacomini; Hippo.: hippocampal; Inf.: inferior; Intralim.: intralimbic; Lat.: lateral; M1.: M1 segment of middle cerebral artery; M2.: M2 segment of middle cerebral artery; Men.: meningeal; Mes.: mesencephalic; Mid.: middle; Parahippo.: parahippocampal; P.C.A.: posterior cerebral artery; Plex.: plexus; Post.: posterior; Semilun.: semilunar; Sulc.: sulcus; Sup.: superior; Temp.: temporal; Tent.: tentorial; Uncin.: uncinate; V.: vein. 
calcarine sulcus. The P4 contains the cortical branches of the PCA.

The P2a is in close relation to the anterior MTR which gives many branches. These branches, the anterior inferior temporal artery, the anterior hippocampal-parahippocampal artery, and the main trunk of P2a, have the main contribution to the arterial supply of the anterior MTR [6].

The P2p is in close relation to the middle MTR. The PCA shows a bifurcation or trifurcation in most hemispheres (in $89 \%$ of hemispheres) at the middle part of this region at a distance of $6.7 \mathrm{~mm}$ (range $0-21 \mathrm{~mm}$ ) to the posterior limit of the uncus. The most common bifurcation pattern is a division into a parietooccipital arterial trunk and a posterior inferior temporal artery. The next common bifurcation pattern is a division into a parietooccipital arterial trunk and an inferior common temporal artery, which then divides into anterior, middle, and posterior inferior temporal arteries (Figures 4(c), 4(d), 6(c)-6(e)). The posterior parahippocampal arteries originating from the posterior inferior temporal artery give the arterial supply to the middle MTR $[6]$.

The P3 and the P4 segments are related to the posterior MTR and do not give any branches to the inferior surface of the temporal lobe. The PCA courses below the isthmus of the cingulate gyrus towards the calcarine sulcus to make its distal bifurcation into the parietooccipital artery and the calcarine artery (Figure 4(c)). The posterior inferior temporal artery, the posterior hippocampal arteries, the splenial artery, the calcarine artery, and the parietooccipital artery are the branches of the PCA emerging at this segment which contribute to the arterial supply of the posterior MTR [6].

3.1.2. Internal Carotid Artery. The C4 segment of the ICA begins where the artery enters the dura and courses superior, posterior, and lateral to its bifurcation into the anterior and middle cerebral arteries. The $\mathrm{C} 4$ is divided into 3 subsegments: the ophthalmic, the communicating, and the choroidal [34]. The first segment after the ICA enters into the intradural space is the ophthalmic segment, which has the origin of the ophthalmic artery at its distal limit. The second segment is the communicating segment, which starts at the origin of the ophthalmic artery and ends at the origin of the PCoA. The third segment is the choroidal segment, which continues from the origin of AChA to the bifurcation of the ICA.

These segments may come into view during a pterional craniotomy or its variations. The surgeon finds the AChA before the PCoA because the AChA is closer to the bifurcation of the ICA, the AChA originates closer to the lateral wall of the ICA than the PCoA, and the AChA follows a more lateral course than the PCoA after their origin.

Fernández-Miranda et al. [6] stated that arterial branches of the ICA, which supply the MTR, were present in $45 \%$ of hemispheres. If present, these arteries always arose from the choroidal segment of the ICA. They suggested that their presence is in close relation to the absence of the branches of the AChA and the MCA.
3.1.3. Anterior Choroidal Artery. The AChA arises from the posterior wall of the ICA. Its origin is nearer to the origin of the PCoA than to the bifurcation of the ICA in most cases. Additionally, it can arise directly from the PCoA [6]. The AChA may show two different types of duplication. It can arise from the posterior wall of the ICA and then immediately divide into two arteries, or the AChA arises as two different trunks from the C4. The AChA is divided into two segments: cisternal and plexal [37]. The cisternal segment follows an initial posteromedial direction in the carotid cistern but then follows a posterior, lateral, and superior direction under the optic tract by staying lateral to it. It follows the superior surface of the posterior segment of the uncus and enters into the ventricle through the choroidal fissure. This entry point into the ventricle is the inferior choroidal point in most cases. Another entry point is also possible a few millimeters posterior to the inferior choroidal point. The length of this segment was measured as $23 \mathrm{~mm}$ and $24 \mathrm{~mm}$ in two different neuroanatomical studies [6, 34].

The cisternal segment of the AChA supplies only the anterior MTR. This arterial supply can be divided into three areas: anterosuperior, medial, and inferior. The anterosuperior area is the anterior segment of the uncus and is supplied by the anterior uncal arteries. The medial area depicts the posterior segment of the uncus and is supplied by the posterior uncal arteries. The inferior area is the entorhinal area, and Fernández-Miranda et al. described that the AChA gives only a minimal contribution to the arterial supply of this area (Figure 6(b)) [6]. The arterial supply of the entorhinal area, which occupies the anterior portion of the parahippocampal gyrus, depends mainly on the PCA and the MCA. The PCA gave the anterior hippocampalparahippocampal arteries and its branches, called the anterior parahippocampal arteries, to the entorhinal area in all cases (Figure 6(e)).

The plexal segment of the AChA begins at the entry point of the artery into the ventricle. This segment mainly gives the arterial supply of the choroid plexus in the temporal horn, but it may also supply the choroid plexus at more posterior levels. This segment gives off one to four perforating branches.

3.1.4. Middle Cerebral Artery. The MCA is divided into four segments (Figures 4(e) and 5(c)) [34]: M1 or the sphenoidal segment, M2 or the insular segment, M3 or the opercular segment, and M4 or the cortical segment. All four segments supply different parts of the temporal lobe. The M1 starts at the origin of the MCA and ends at the genu. The M2 segment consists of the arteries lying on the insular surface. The M3 is the segment within the sylvian cistern starting at the circular sulcus of the insula to end at the surface of the sylvian fissure. The M4 consists of cortical branches at the lateral convexity.

The bifurcation of the MCA occurs just proximal to the end of the M1; thus, the M1 is divided into prebifurcation and postbifurcation parts. Two other important vascular structures arise from the M1 other than the superior and inferior trunks after the bifurcation. The cortical branches arising from the M1 proximal to the bifurcation are referred to as early branches. Early branches may reach to the frontal 


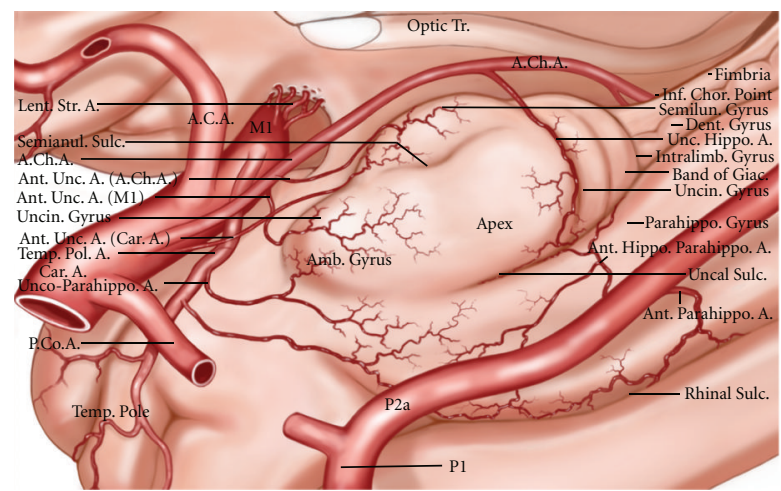

(a)

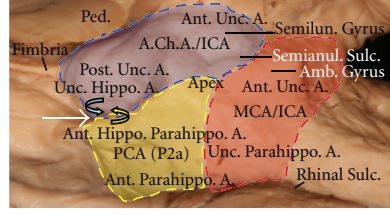

(b)

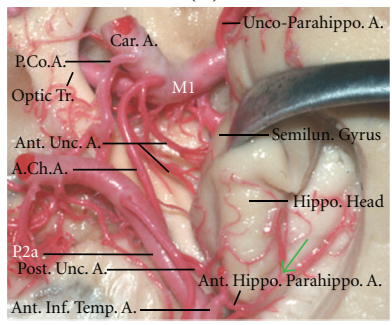

(d)

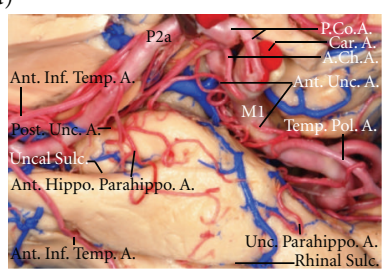

(c)

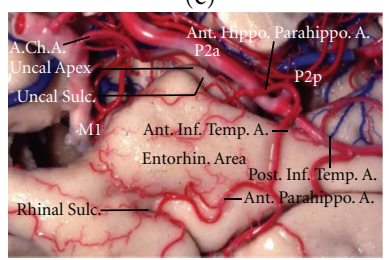

(e)

FIGURE 6: (a) The medial view of the anterior segment of the medial temporal region (MTR). (This illustration correlates with Figures 6(b) and $6(\mathrm{c})$ ). The anterior choroidal artery (AChA) gives off an anterior uncal artery that irrigates the semilunar gyrus and an uncohippocampal artery that irrigates the uncinate gyrus and band of Giacomini and penetrates the uncal sulcus to vascularize the extraventricular hippocampal head. The internal carotid artery (ICA) gives off an anterior uncal artery that supplies the semilunar gyrus. This branch usually is present when the anterior uncal artery of the AChA is absent. An anterior uncal artery also arises from the M1 segment of the middle cerebral artery (MCA) and supplies the ambient gyrus. An unco-parahippocampal artery arises from the temporopolar artery and irrigates both the ambient gyrus and the anterior parahippocampal area. Branches from the P2a segment of the PCA irrigate the anterior parahippocampal region (anterior parahippocampal artery) or both the anterior parahippocampal gyrus and hippocampal head (anterior hippocampal-parahippocampal artery). (b) The medial surface of the anterior segment of the left MTR. The white arrow points the posterior end of the uncal notch. The anterior part of this segment is irrigated by middle cerebral branches (orange shaded area), the posterosuperior part is supplied by anterior choroidal branches (blue shaded area), and the posteroinferior part is vascularized by posterior cerebral branches (yellow shaded area). The ICA typically supplies the area supplied by the AChA and the MCA if their branches are absent. The branches of the MCA are the anterior uncal artery superiorly and the unco-parahippocampal artery inferiorly. The branches of the AChA are the anterior uncal artery anteriorly, the posterior uncal artery posteriorly, and the unco-hippocampal artery posteroinferiorly. The branches of the PCA are the anterior hippocampal-parahippocampal artery medially and the anterior parahippocampal artery laterally. Areas of vascular anastomosis are typically found at the confluence of vascular territories (curved arrows). (c) The same view of (a) in a silicon injected anatomic specimen. (d) Inferior view of the anterior segment of the left MTR. The inferior lip of the posterior uncal segment has been removed to expose the extraventricular hippocampal head. The semilunar gyrus has been retracted to expose the branches of the AChA. Two anterior uncal arteries arise from the first one-third of the AChA and irrigate the semilunar gyrus. A posterior uncal artery from the AChA penetrates the uncal sulcus and irrigates the extraventricular hippocampal head. An anterior hippocampal-parahippocampal artery arising from the anteroinferior temporal branch of the PCA gives rise to an anterior hippocampal branch that supplies the extraventricular hippocampal head and anastomoses with the unco-hippocampal branch of the AChA (green arrow). (e) Lower surface of the anterior segment of the right MTR. The entorhinal area is irrigated medially by the parahippocampal branch of the anterior hippocampal-parahippocampal artery that arises from the P2a, and laterally by a large anterior parahippocampal artery that originates from the anterior inferior temporal artery. A.: artery; A.C.A.: anterior cerebral artery; A.Ch.A.: anterior choroidal artery; Amb.: ambient; Ant.: anterior; Car.: carotid; Chor.: choroidal; Dent.: dentate; Entorhin.: entorhinal; Giac.: Giacomini; Hippo.: hippocampus; ICA: internal carotid artery; Inf.: inferior; Intralimb.: intralimbic; Lent.: lenticulo; M.C.A.: middle cerebral artery; M1.: M1 segment of middle cerebral artery; Parahippo.: parahippocampal; P.C.A.: posterior cerebral artery; P2A.: anterior part of the P2 segment of posterior cerebral artery; P2P.: posterior part of the P2 segment of posterior cerebral artery; P.Co.A.: posterior communicating artery; Ped.: peduncle; Pol.: polar; Semianul.: semiannular; Semilun.: semilunar; Str.: striate; Sulc.: sulcus; Temp.: temporal; Tr.: tract; Unc.: uncal; Uncin.: uncinate; V.: vein. 
or the temporal lobes with the temporopolar area being the most common temporal area supplied by an early branch [38]. The M1 supports the arterial supply of the MTR with 1 to 3 branches in $94 \%$ of the specimens. Fernández-Miranda et al. further detailed this data. They described that these branches originate from the main M1 trunk in $42 \%$ of the cases, from a temporopolar artery in $41 \%$ of the cases, and from an early branch other than temporopolar artery in $14 \%$ of the cases [6].

Three types of the distribution of the MCA branches can be identified. The anterior group, which supplies the anterior segment of the uncus, was named as the anterior uncal arteries. The anteroinferior group, which has the distribution to the anterior entorhinal area, was named as the uncoparahippocampal arteries. These two groups contain $92 \%$ of all branches directed to the MTR from the MCA. The third group of arteries, which gives supply to the anterior part of the entorhinal area, was named as the anterior parahippocampal arteries. While anterior uncal arteries arise mostly from the main M1 trunk, the unco-parahippocampal arteries and anterior parahippocampal arteries arise mainly from the temporopolar artery [6].

The arteries at the superior surface of the temporal lobe belong to the opercular segment (M3), which come into view during the splitting of the sylvian fissure; the cortical branches at the lateral surface belong to the M4 which are grouped into distribution areas as, from anterior to posterior, the temporopolar, anterior, middle, and posterior temporal areas [34].

3.2. Venous Drainage. The venous drainage of the cerebrum is divided into a superficial group and a deep group [34]. This also applies to the temporal lobe. The microvascular anatomy of the venous drainage of the cerebrum was described in other reports $[39,40]$, and the venous drainage of the sylvian fissure was further detailed by Tanriover et al. [4].

Two most important venous structures at the lateral surface of the temporal lobe are the superficial sylvian vein (SSV) and the vein of Labbe. The SSV usually arises as a single trunk at the most posterior end of the sylvian fissure but may also start as two trunks that join together before their drainage. Tanriover et al. described that the SSV emptied into the sphenoparietal sinus in 35 of 43 hemispheres [4]. In the remaining hemispheres, the SSV drained directly into the cavernous sinus or into a sphenopetrosal sinus. In their study, the mean distance between the limen insula and the junction of the SSV with the sphenoparietal sinus is measured $24.8 \mathrm{~mm}$. The vein of Labbe is the largest vein on the lateral surface of the temporal lobe that connects the SSV and the transverse sinus and is located in the drainage area of the middle temporal vein in more than half of the hemispheres [39]. The SSV and the vein of Labbe may be hypoplastic or absent. The most consistent dominant superficial anastomotic veins are the SSV and the vein of Labbe followed by vein of Labbe and the vein of Trolard [41].

Oka et al. divided the superficial venous anatomy of the temporal lobe into a lateral group and an inferior group $[4,39]$. The lateral group of veins is further divided into two additional groups: an ascending group consists of temporosylvian veins that drain towards the sylvian fissure and a descending group consists of anterior, middle, and posterior temporal veins that drain into the tentorial sinuses. Tanriover et al. stated that there are a mean of four temporosylvian veins draining into SSV [4]. These veins drain the temporopolar area and the superior temporal gyrus anterior to the posterior limit of the sylvian fissure. Also, temporosylvian veins have a minor role at the venous drainage of the inferior limiting sulcus and the posterior long gyrus of the insula. The anterior temporal vein collects the venous drainage from the anterior one-third of the lateral surface except the superior temporal gyrus and drains mostly into a tentorial sinus. The middle temporal vein drains the midportion of the lateral surface and empties into the transverse sinus, a tentorial sinus, or the vein of Labbe. The posterior temporal vein drains the posterior third of the lateral convexity of the temporal lobe and courses in a nearly vertical route to drain into a tentorial sinus or less commonly into the vein of Labbe.

The lateral part of the inferior surface of the temporal lobe is drained via the anterior, middle, and posterior temporobasal veins. They empty into tentorial sinuses.

The deep middle cerebral vein and the venous drainage of the MTR empty into the deep venous system. The deep venous system begins with the basal vein which has three segments [42] (Figures 7(a)-7(c)). The deep middle cerebral vein is formed by the junction of the insular veins at the level of limen insula [4]. The first (striate) segment is composed of the deep middle cerebral vein and inferior striate veins that unify to form the basal vein. The joining of the anterior cerebral, olfactory, and frontoorbital veins completes the striate segment, which courses posterior at the upper part of the anterior segment of the uncus to meet with the peduncular vein at the apex of the uncus. The second (peduncular) segment begins at this point to course posterior in the crural cistern. The peduncular segment is divided into an anterior part and a posterior part. The anterior part of the peduncular segment is referred to as the anterior basal anastomotic vein, because it joins the striate segment and the posterior part of the peduncular segment. The posterior peduncular segment starts where the inferior ventricular vein joins the basal vein and finishes where the lateral mesencephalic vein joins the basal vein. The third (mesencephalic) segment is also known as the posterior anastomotic vein, since it unifies the peduncular segment with the vein of Galen.

The anterior MTR has relationship with the striate segment and the anterior peduncular segment. This region shows mainly two types of drainage. If typical variant is present, the venous drainage empties into the posterior peduncular segment via the anterior basal anastomotic vein. Fernández-Miranda et al. found this variant in 23 of 37 cases [6]. The other variant is present if there is no anastomosis between the striate segment and the posterior peduncular segment. In this group, the venous drainage of the anterior MTR emptied into the cavernous sinus or into the sphenoparietal sinus via a large preuncal vein. 

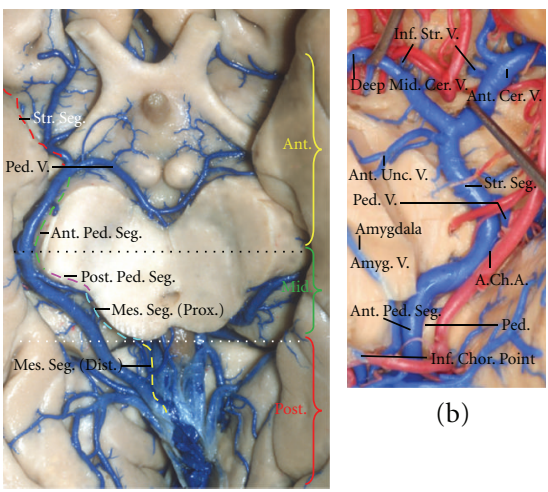

(b)

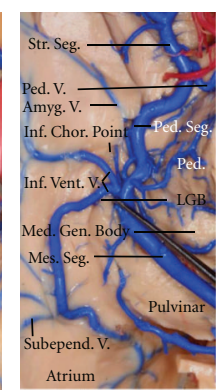

(c)

(a)

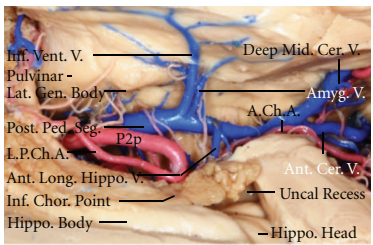

(d)

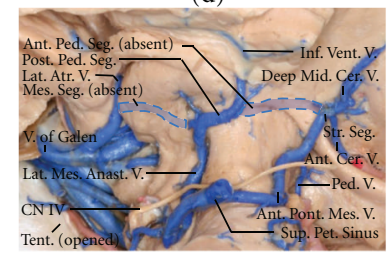

(f)

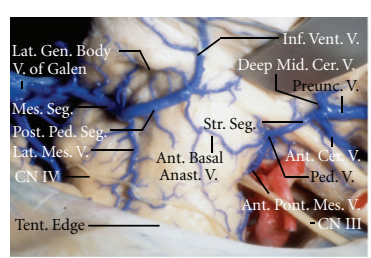

(e)

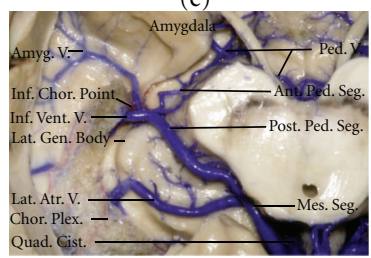

(g)

Figure 7: (a) Inferior view. The basal vein and its tributaries have been exposed. The segmental classification of the basal vein (right hemisphere) and the segmental classification of the MTR (left hemisphere) are shown. The striate (red interrupted line) and anterior peduncular (green interrupted line) segments of the basal vein are located along the anterior MTR (yellow bracket). The posterior peduncular (purple interrupted line) and proximal mesencephalic (aqua interrupted line) segments of the basal vein are situated in the middle MTR (green bracket). The distal mesencephalic segment (yellow interrupted line) of the basal vein belongs to the posterior MTR (red bracket). The anterior peduncular segment is also referred to as the anterior anastomotic vein, because it allows the striate segment to communicate with the posterior peduncular segment. The mesencephalic segment of the basal vein also is referred to as the posterior basal anastomotic vein because it connects the peduncular segment to the vein of Galen. (b) Basal view of the striate and anterior peduncular segments of the basal vein. The part of the amygdala below the level of the optic tract has been removed. The carotid and crural cisterns have been opened to expose the striate and anterior peduncular segments of the basal vein. The anterior and deep medial cerebral veins meet to form the striate segment of the basal vein. The anterior peduncular segment begins where the peduncular vein joins the basal vein, and the posterior peduncular segment starts at the junction with the inferior ventricular vein. (c) Inferior view of the roof of the temporal horn in another right hemisphere. The junctional point between the inferior ventricular vein and the peduncular segment corresponds to the cisternal level of the inferior choroidal point and the junction of the crural and ambient cisterns. Several large subependymal veins draining the roof and lateral wall of the temporal horn join to form the inferior ventricular vein. (d) The right temporal horn has been opened, and the ambient and crural cistern have been exposed by opening the choroidal fissure. The amygdalar vein drains separately from the inferior ventricular vein into the basal vein. The anterior longitudinal hippocampal vein empties into the basal vein anterior to the inferior ventricular vein. (e) Lateral view of the right tentorial incisura. The temporal lobe has been removed to expose the basal vein. The anterior peduncular segment of the basal vein is hypoplastic. Failure of anastomosis between the striate and peduncular segments of the basal vein results in formation of a prominent preuncal vein that drains forward into the sphenoparietal sinus. (f) Same view as (e) in a different specimen. Both the anterior peduncular (anterior anastomotic vein) and the mesencephalic segment (posterior anastomotic veins; blue shaded areas) are absent, and the striate, peduncular, and mesencephalic segments of the basal vein are disconnected from each other. The striate segment drains inferiorly via the anterior pontomesencephalic vein, the peduncular segment drains inferiorly through a prominent lateral mesencephalic vein, and the tributaries of the mesencephalic segment drain directly into the vein of Galen. (g) Inferior view of the right basal vein. The anterior peduncular segment (anterior anastomotic vein) of the basal vein is hypoplastic. The inferior ventricular vein empties into and forms the posterior peduncular and mesencephalic segments of the basal vein, and the anterior segment of the basal vein drains anteriorly. A prominent lateral atrial vein drains into the mesencephalic segment. A.Ch.A.: anterior choroidal artery; Amyg.: amygdalar; Anast.: anastomotic; Ant.: anterior; Atr.: atrial; Cer.: cerebral; Chor.: choroidal; cist.: cistern; CNIII.: oculomotor nerve; CNIV.: trochlear nerve; Dist.: distal; Gen.: geniculate; Hippo.: hippocampus; Inf.: inferior; Lat.: lateral; Long.: longitudinal; Med.: medial; Mes.: mesensephalic; Mid.: middle; LGB:: lateral geniculate body; L.P.Ch.A.: lateral posterior choroidal artery; Ped.: peduncle; Plex.: plexus; Pon.: ponto; Post.: posterior; Preunc.: preuncal; Prox.; proximal; Seg.: segment; Str.: striate; Subepend.: subependimal; Quad.: quadrigeminal; Tent.: tentorial; Unc.: uncal; V.: vein; Vent.: ventricular. 
The middle MTR is in close relation with the posterior peduncular segment and the proximal part of the mesencephalic segment. The inferior ventricular vein drains the roof and the lateral wall of the temporal horn as well as the anterior part of optic radiations. In the absence or hypoplasia of the peduncular segment, the inferior ventricular vein forms the second segment of the basal vein. Other tributaries to the second segment of the basal vein are anterior hippocampal vein, which drains the extraventricular head of hippocampus, and anterior longitudinal hippocampal vein, which drains the body of the hippocampus (Figures 7(a), $7(\mathrm{~d})-7(\mathrm{~g}))$.

The posterior MTR is in close relation with the distal part of the mesencephalic segment of the basal vein, which courses in the quadrigeminal cistern and drains into the vein of Galen or the internal cerebral vein. The tributaries from this region into the basal vein are the posterior longitudinal hippocampal vein, medial temporal vein, lateral and medial atrial veins. The posterior longitudinal hippocampal vein takes the drainage of the tail of hippocampus. The medial temporal vein drains the inferior surface of the posterior part of the parahippocampal gyrus.

\section{Discussion}

Although thousands of patients with temporal lobe epilepsy were operated, there is still controversy regarding the best surgical approach $[8,10,43,44]$. The main goal is longterm seizure free outcome while avoiding functional deficits. The main risks for functional decline are visual deficit or neurocognitive damage, which may be related to the choice of operative route to the medial temporal lobe. Different surgical methods will be discussed with these concerns in mind.

Surgical methods for temporal lobe epilepsy can be divided in three groups from a neuroanatomical view: lateral approaches, inferior approaches, transsylvian approaches [14]. The lateral approaches are the anterior temporal lobectomy (ATL) and the transcortical selective amygdalohippocampectomy (TCAH); the inferior approaches are the subtemporal approach and the transparahippocampal approach; and the transsylvian approaches are the transsylvian selective amygdalohippocampectomy (SelAH) and the transcisternal approach. In this chapter, the ATL, TCAH, and the SelAH will be discussed. The subtemporal approach [45], the transparahippocampal approach [46], and the transcisternal approach [47] will not be discussed since they are performed only in selected centers.

4.1. Neocortical Removal and Approach to the Temporal Horn. After sufficient exposure of the lateral surface of the temporal lobe, the sulcal and vascular anatomy should be inspected carefully. The arterial structures in view are the M4 segment of the MCA and divided into the temporopolar area and anterior, middle, and posterior temporal areas. The temporopolar area can be supplied occasionally by an early branch of the MCA [38]. The location of the vein of Labbe should also be noted. This vein is located at the drainage area of the middle temporal vein in most cases [39], and care should be taken to preserve it, since it is commonly the dominant superficial anastomotic vein [41].

After the neocortical removal, the temporal horn comes into view which has the collateral eminence at its floor. The collateral eminence is the indentation of the collateral sulcus towards the temporal horn and lies lateral to the hippocampus [34]. The neural tissue lateral to the collateral eminence can be removed safely without any risk of damaging midbrain structures. Another reliable landmark to achieve a safe neocortical resection is the tentorial edge (Figure 5(a)). As long as the resection is aimed lateral to the free edge of the tentorium, the damage to the inferior limiting sulcus, the sylvian fissure, and midbrain structures will be avoided.

Clinical data suggests that the ATL causes high risk of damaging optic radiations. Yeni et al. stated that this risk is higher during the ATL comparing to the TSAH [9]. The neuroanatomical data support these findings. The Meyer's loop was found to cover the roof of temporal horn and to exceed the anterior wall of the temporal horn a few millimeters, thus opening the ventricle at the superior wall may damage optic radiations [12]. Additionally, Meyer's loop was found an average of $31.4 \mathrm{~mm}$ posterior from the temporal pole [13]. Therefore, any resection posterior to this extent carries the risk of causing damage to optic radiations.

The neocortical removal can also lead to negative neurocognitive sequelae. The ILF connects anterior parts of the temporal lobe to the fusiform gyrus and the occipital lobe. The neocortical resection may include the ILF itself or the cortical areas it originates. And since the areas the ILF connects are thought to have role in learning and remembering of visual stimuli [22], the neocortical resection can cause memory problems.

A cortical incision to the middle temporal gyrus or deep to the superior temporal sulcus is made at the TCAH to reach to the ventricle through its lateral wall. Olivier described that the cortical incision is made to the middle temporal gyrus rather than the superior temporal sulcus [48]. Although transsulcal approach has less distance to reach to the temporal horn, the need for the retraction of the sulcal lips and possible presence of vascular structures deep to sulcus makes this incision demanding. The difficulty of this technique is to find the temporal horn, because a blind dissection in the white matter can lead to significant deficits. Wen et al. described a very useful technique to find the temporal horn [5]. The TCAH carries the risk of damaging both optic radiations and the ILF. But the risk of damaging optic radiations is less than the risk with the ATL, since optic radiations do not cover the anterior portion of the lateral wall of the temporal horn [12].

The TSAH, described by Wieser and Yasargil, is performed with a different route to the temporal horn. The incision is made near to the inferior limiting sulcus after the sylvian fissure is opened [10]. This incision is placed at the temporal stem between the temporopolar artery and anterior temporal artery and carried back $15-20 \mathrm{~mm}$ from the limen insula. This approach has its own risks to cause functional deficits by damaging optic radiations, the UF, and the IOFF. 
The distance of the LGB to the limen insula is on average $25.1 \mathrm{~mm}$, and there is no clear demarcation between the LGB and the thalamus. During the incision to the inferior limiting sulcus, the thalamus or the LGB can be harmed. Choi et al. stated that it is impossible to avoid the optic radiations if the incision is carried $15-20 \mathrm{~mm}$ posterior to the limen insula. Optic radiations can be avoided at a level $10 \mathrm{~mm}$ posterior to the limen insula, if the dissection is made between 7 and 25 degrees medially from the sagittal plane, while damaging the mesencephalon and diencephalic structures is highly possible with such dissection. They described that an incision straight inferiorly to the limen insula and to the following $5 \mathrm{~mm}$ of the inferior limiting sulcus avoids Meyer's loop [13]. Although the anatomy is more complicated with the TSAH, the risk of damaging optic radiations is lower than the risk ATL carries [9].

It is impossible to preserve the UF with an incision extending posterior from the limen insula. The destruction of this white matter bundle can be the cause of the memory deficits occurring after TSAH, because it is stated that the UF functions at recognizing faces and objects by connecting the orbitofrontal and temporopolar areas [18-20].

The IOFF is another important white matter structure coursing in the temporal stem. Its average distance to limen insula was found $10.9 \mathrm{~mm}$ [49]. The incision to the inferior limiting sulcus can cause damage to the IOFF and produce semantic paraphasias [28].

4.2. Hippocampectomy. The hippocampectomy begins with the opening of the choroidal fissure beginning at the inferior choroidal point anteriorly. The AChA enters into the temporal horn through the inferior choroidal point or just a few millimeters posterior to it. The inferior choroidal point is also the intraventricular representation of the posterior limit of the uncus and determines the border between anterior and middle MTR. The head of the hippocampus is situated anterior to inferior choroidal point, thus located in the anterior MTR. The body of the hippocampus located posterior to this point is in the middle MTR. Since the choroid plexus is attached to the choroidal fissure, the choroid plexus is related only to the body of the hippocampus. The choroid plexus originates from the tela choroidea, which is attached to fimbria of the fornix and to the thalamus at the edges of the fissure. The tela attached to the fimbria and thalamus is called the taenia fimbriae and taenia thalami.

The choroidal fissure should be opened on the forniceal side to avoid damage to the diencephalic structures and to the vascular structures, such as the AChA, lateral posterior choroidal arteries, and the inferior ventricular vein, by leaving them at the thalamic side (Figures 4(a), 5(b), and $7(\mathrm{~g}))$. Once the anterior part of the choroidal fissure is opened, the PCA comes into view under the arachnoidal membrane of the ambient cistern. One should note that the PCA is related to the inferior part of the posterior segment of the uncus. The parahippocampal gyrus coursing medially throughout the ambient cistern can be seen once the opening of the choroidal fissure is extended posterior. At this point, a prominence on the thalamic side, the pulvinar, can be marked.
The next step is the anterior and medial disconnection of the head of hippocampus via the uncal recess that leads the surgeon to the apex of the uncus. The oculomotor nerve located medial to the apex of the uncus and the P2a located inferomedial to the posterior segment of the uncus may come into the view under the arachnoid membrane. This view shows that the inferior part of the posterior segment of the uncus is also removed.

The medial disconnection via the choroidal fissure follows the anterior disconnection. After the choroidal fissure is opened, an arachnoid membrane adjacent to the hippocampus is met before reaching the arachnoid membrane of the crural and the ambient cisterns. The hippocampal branches from the AChA and the PCA enter through this membrane. The medial disconnection will be carried out by separating the structures of the MTR from these arteries by following a direction towards the inferior surface of the parahippocampal gyrus until reaching the collateral sulcus.

The last step is the posterior disconnection. The posterior limit of the tail of hippocampus is where he tail meets the calcar avis, which is the inferior prominence at the medial wall of the atrium.

4.3. Amygdalectomy. The temporal amygdala is located entirely in the anterior segment of the uncus. The removal of the anterior segment of the uncus exposes the ICA and the proximal M1. The oculomotor nerve and the PCA have already been exposed during the medial disconnection of the head of the hippocampus.

Resection of the superior part of the uncus carries risk of damaging the globus pallidus unintentionally, since there is no clear border between the globus pallidus and the amygdala. The proximal part of the cisternal segment of the AChA is related to the superior surface of the anterior segment of the uncus in $68 \%$ of the hemispheres and the distal part of the cisternal segment of the AChA is related to the superior surface of the posterior segment of the uncus in $86 \%$ of the hemispheres [1]. Since this relationship is not present in every case, the AChA itself is not a reliable landmark to determine the superior limit of resection of the uncus and the amygdala.

Wen et al. described an imaginary line starting at the ICA bifurcation or the proximal M1 and ending at the inferior choroidal point (carotid-choroidal point) important in avoiding extension of the amygdala resection in the globus pallidus, but add that the optic tract may also be useful to determine the superior extent of the amygdala removal in TSAH (Figure 4(b)) [1].

\section{Conclusion}

While the main goal in epilepsy surgery is long-term seizure control, avoidance of motor, visual, and cognitive deficits helps to optimize an improved quality of life for these patients. Despite advances in image guided neurosurgical navigation, a three-dimensional knowledge of the microsurgical anatomy is the best resource for precise TLE surgery. A thorough knowledge of the microanatomy of this region leads epilepsy surgeon to a better understanding of 
the functional anatomy, and a better appreciation of predicted results from different surgical approaches.

\section{References}

[1] H. T. Wen, A. L. Rhoton Jr., E. De Oliveira et al., "Microsurgical anatomy of the temporal lobe: part 1: mesial temporal lobe anatomy and its vascular relationships as applied to amygdalohippocampectomy," Neurosurgery, vol. 45, no. 3, pp. 549-592, 1999.

[2] M. Ono, S. Kubik, and C. D. Abernathey, Atlas of the Cerebral Sulci, Thieme, Stuttgart, Germany, 1990.

[3] H. T. Wen, A. L. Rhoton Jr., E. De Oliveira, L. H. M. Castro, E. G. Figueiredo, and M. J. Teixeira, "Microsurgical anatomy of the temporal lobe: part 2-sylvian fissure region and its clinical application," Neurosurgery, vol. 65, no. 6, supplement, pp. ons1-ons35, 2009.

[4] N. Tanriover, A. L. Rhoton Jr., M. Kawashima, A. J. Ulm, and A. Yasuda, "Microsurgical anatomy of the insula and the sylvian fissure," Journal of Neurosurgery, vol. 100, no. 5, pp. 891922,2004

[5] H. T. Wen, A. L. Rhoton Jr., and R. Marino Jr., "Gray matter overlying anterior basal temporal sulci as an intraoperative landmark for locating the temporal horn in amygdalohippocampectomies," Neurosurgery, vol. 59, no. 4, supplement 2, pp. 221-227, 2006.

[6] J. C. Fernández-Miranda, E. De Oliveira, P. A. Rubino, H. T. Wen, and A. L. Rhoton, "Microvascular anatomy of the medial temporal region: part 1: its application to arteriovenous malformation surgery," Neurosurgery, vol. 67, no. 3, supplement 1, pp. ons237-ons276, 2010.

[7] T. Tanriverdi, R. W. R. Dudley, A. Hasan et al., "Memory outcome after temporal lobe epilepsy surgery: corticoamygdalohippocampectomy versus selective amygdalohippocampectomy," Journal of Neurosurgery, vol. 113, no. 6, pp. 1164-1175, 2010.

[8] T. Tanriverdi, A. Olivier, N. Poulin, F. Andermann, and F. Dubeau, "Long-term seizure outcome after mesial temporal lobe epilepsy surgery: corticalamygdalohippocampectomy versus selective amygdalohippocampectomy," Journal of Neurosurgery, vol. 108, no. 3, pp. 517-524, 2008.

[9] S. N. Yeni, N. Tanriover, O. Uyanik et al., "Visual field defects in selective amygdalohippocampectomy for hippocampal sclerosis: the fate of meyers loop during the transsylvian approach to the temporal horn," Neurosurgery, vol. 63, no. 3, pp. 507$513,2008$.

[10] H. G. Wieser and M. G. Yasargil, "Selective amygdalohippocampectomy as a surgical treatment of mesiobasal limbic epilepsy," Surgical Neurology, vol. 17, no. 6, pp. 445-457, 1982.

[11] M. G. Yaşargil, N. Krayenbühl, P. Roth, S. P.C. Hsu, and D. C.H. Yaşargil, "The selective amygdalohippocampectomy for intractable temporal limbic seizures," Journal of Neurosurgery, vol. 112, no. 1, pp. 168-185, 2010.

[12] P. A. Rubino, A. L. Rhoton Jr., X. Tong, and E. De Oliveira, "Three-dimensional relationships of the optic radiation," Neurosurgery, vol. 57, no. 4, supplement, pp. S-219-S-227, 2005.

[13] C. Choi, P. A. Rubino, J. C. Fernandez-Miranda, H. Abe, and A. L. Rhoton Jr., "Meyer's loop and the optic radiations in the transsylvian approach to the mediobasal temporal lobe," Neurosurgery, vol. 59, no. 4, supplement 2, pp. 228-235, 2006.

[14] E. H. Sincoff, Y. Tan, and S. I. Abdulrauf, "White matter fiber dissection of the optic radiations of the temporal lobe and implications for surgical approaches to the temporal horn," Journal of Neurosurgery, vol. 101, no. 5, pp. 739-746, 2004.
[15] K. B. Mahaney and S. I. Abdulrauf, "Anatomic relationship of the optic radiations to the atrium of the lateral ventricle: description of a novel entry point to the trigone," Neurosurgery, vol. 63, no. 4, supplement 2, pp. ONS195-ONS203, 2008.

[16] J. C. Fernández-Miranda, A. L. Rhoton Jr., Y. Kakizawa, C. Choi, and J. Álvarez-Linera, "The claustrum and its projection system in the human brain: a microsurgical and tractographic anatomical study - laboratory investigation," Journal of Neurosurgery, vol. 108, no. 4, pp. 764-774, 2008.

[17] H. Duffau, P. Gatignol, S. Moritz-Gasser, and E. Mandonnet, "Is the left uncinate fasciculus essential for language? : aa cerebral stimulation study," Journal of Neurology, vol. 256, no. 3, pp. 382-389, 2009.

[18] C. Papagno, C. Miracapillo, A. Casarotti et al., "What is the role of the uncinate fasciculus? surgical removal and proper name retrieval," Brain, vol. 134, no. 2, pp. 405-414, 2011.

[19] T. J. Grabowski, H. Damasio, D. Tranel, L. L. Boles Ponto, R. D. Hichwa, and A. R. Damasio, "A role for left temporal pole in the retrieval of words for unique entities," Human Brain Mapping, vol. 13, no. 4, pp. 199-212, 2001.

[20] A. M. Proverbio, S. Lilli, C. Semenza, and A. Zani, "Erp indexes of functional differences in brain activation during proper and common names retrieval," Neuropsychologia, vol. 39, no. 8, pp. 815-827, 2001.

[21] M. Catani, R. J. Howard, S. Pajevic, and D. K. Jones, "Virtual in vivo interactive dissection of white matter fasciculi in the human brain," Neuroimage, vol. 17, no. 1, pp. 77-94, 2002.

[22] L. Cohen, S. Dehaene, L. Naccache et al., "The visual word form area. spatial and temporal characterization of an initial stage of reading in normal subjects and posterior split-brain patients," Brain, vol. 123, no. 2, pp. 291-307, 2000.

[23] M. Catani, D. K. Jones, R. Donato, and D. H. Ffytche, "Occipito-temporal connections in the human brain," Brain, vol. 126, no. 9, pp. 2093-2107, 2003.

[24] N. Makris, D. N. Kennedy, S. McInerney et al., "Segmentation of subcomponents within the superior longitudinal fascicle in humans: a quantitative, in vivo, dt-mri study," Cerebral Cortex, vol. 15, no. 6, pp. 854-869, 2005.

[25] J. C. Fernández-Miranda, A. L. Rhoton Jr., J. Álvarez-Linera, Y. Kakizawa, C. Choi, and E. P. De Oliveira, "Three-dimensional microsurgical and tractographic anatomy of the white matter of the human brain," Neurosurgery, vol. 62, no. 6, supplement 3, pp. SHC989-SHC1026, 2008.

[26] M. Catani, D. K. Jones, and D. H. Ffytche, "Perisylvian language networks of the human brain," Annals of Neurology, vol. 57, no. 1, pp. 8-16, 2005.

[27] G. Hickok and D. Poeppel, "Towards a functional neuroanatomy of speech perception," Trends in Cognitive Sciences, vol. 4, no. 4, pp. 131-138, 2000.

[28] H. Duffau, P. Gatignol, E. Mandonnet, P. Peruzzi, N. TzourioMazoyer, and L. Capelle, "New insights into the anatomofunctional connectivity of the semantic system: a study using cortico-subcortical electrostimulations," Brain, vol. 128, no. 4, pp. 797-810, 2005.

[29] G. Hickok and D. Poeppel, "Dorsal and ventral streams: a framework for understanding aspects of the functional anatomy of language," Cognition, vol. 92, no. 1-2, pp. 67-99, 2004.

[30] J. Martino, C. Brogna, S. G. Robles, F. Vergani, and H. Duffau, "Anatomic dissection of the inferior fronto-occipital fasciculus revisited in the lights of brain stimulation data," Cortex, vol. 46, no. 5, pp. 691-699, 2010. 
[31] G. Hickok and D. Poeppel, "The cortical organization of speech processing," Nature Reviews Neuroscience, vol. 8, no. 5, pp. 393-402, 2007.

[32] U. Ebeling and D. Von Cramon, "Topography of the uncinate fascicle and adjacent temporal fiber tracts," Acta Neurochirurgica, vol. 115, no. 3-4, pp. 143-148, 1992.

[33] C. Y. Choi, S. R. Han, G. T. Yee, and C. H. Lee, "A understanding of the temporal stem," Journal of Korean Neurosurgical Society, vol. 47, no. 5, pp. 365-369, 2010.

[34] A. L. Rhoton Jr., “The cerebrum,” Neurosurgery, vol. 51, no. 4, pp. 1-51, 2002.

[35] L. Heimer, G. W. VanHoesen, M. Trimble et al., Anatomy of Neuropsychiatry The New Anatomy of the Basal Forebrain and Its Implications for Neuropsychiatric Illness, Elsevier, Burlington, Mass, USA, 2007.

[36] A. A. Zeal and A. L. Rhoton Jr., "Microsurgical anatomy of the posterior cerebral artery," Journal of Neurosurgery, vol. 48, no. 4, pp. 534-559, 1978.

[37] A. L. Rhoton Jr., K. Fujii, and B. Fradd, "Microsurgical anatomy of the anterior choroidal artery," Surgical Neurology, vol. 12, no. 2, pp. 171-187, 1979.

[38] N. Tanriover, M. Kawashima, A. L. Rhoton Jr., A. J. Ulm, and R. A. Mericle, "Microsurgical anatomy of the early branches of the middle cerebral artery: morphometric analysis and classification with angiographic correlation," Journal of Neurosurgery, vol. 98, no. 6, pp. 1277-1290, 2003.

[39] K. Oka, A. L. Rhoton Jr., M. Barry, and R. Rodriguez, "Microsurgical anatomy of the superficial veins of the cerebrum," Neurosurgery, vol. 17, no. 5, pp. 711-748, 1985.

[40] M. Ono, A. L. Rhoton Jr., D. Peace, and R. J. Rodriguez, "Microsurgical anatomy of the deep venous system of the brain," Neurosurgery, vol. 15, no. 5, pp. 621-657, 1984.

[41] T. Tanriverdi, H. Al-Jehani, N. Poulin, and A. Olivier, "Superficial anastomotic veins: neurosurgical view depending on 251 craniotomies," Canadian Journal of Neurological Sciences, vol. 36, no. 1, pp. 65-71, 2009.

[42] Y. P. Huang and B. S. Wolf, "The basal cerebral vein and its tributaries," in Radiology of the Skull and Brain, T. H. Newton and D. G. Potts, Eds., pp. 2111-2154, CV Mosby, Saint Louis, Mo, USA, 1971.

[43] H. G. Wieser, M. Ortega, A. Friedman, and Y. Yonekawa, "Long-term seizure outcomes following amygdalohippocampectomy," Journal of Neurosurgery, vol. 98, no. 4, pp. 751-763, 2003.

[44] M. T. Lutz, H. Clusmann, C. E. Elger, J. Schramm, and C. Helmstaedter, "Neuropsychological outcome after selective amygdalohippocampectomy with transsylvian versus transcortical approach: a randomized prospective clinical trial of surgery for temporal lobe epilepsy," Epilepsia, vol. 45, no. 7, pp. 809-816, 2004.

[45] T. Hori, S. Tabuchi, M. Kurosaki et al., "Subtemporal amygdalohippocampectomy for treating medically intractable temporal lobe epilepsy," Neurosurgery, vol. 33, no. 1, pp. 50-57, 1993.

[46] T. S. Park, B. F. D. Bourgeois, D. L. Silbergeld, and W. E. Dodson, "Subtemporal transparahippocampal amygdalohippocampectomy for surgical treatment of mesial temporal lobe epilepsy: technical note," Journal of Neurosurgery, vol. 85, no. 6, pp. 1172-1176, 1996.

[47] P. Vajkoczy, K. Krakow, S. Stodieck, B. Pohlmann-Eden, and P. Schmiedek, "Modified approach for the selective treatment of temporal lobe epilepsy: transsylvian-transcisternal mesial en bloc resection," Journal of Neurosurgery, vol. 88, no. 5, pp. 855862,1998 .
[48] A. Olivier, "Transcortical selective amygdalohippocampectomy in temporal lobe epilepsy," Canadian Journal of Neurological Sciences, vol. 27, supplement 1, pp. S68-S76, 2000.

[49] J. Martino, F. Vergani, S. G. Robles, and H. Duffau, "New insights into the anatomic dissection of the temporal stem with special emphasis on the inferior fronto-occipital fasciculus: implications in surgical approach to left mesiotemporal and temporoinsular structures," Neurosurgery, vol. 66, no. 3, supplement, pp. 4-12, 2010. 


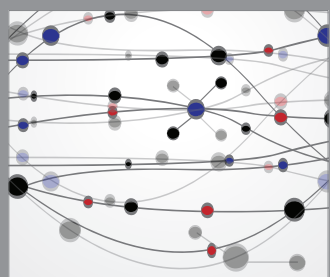

The Scientific World Journal
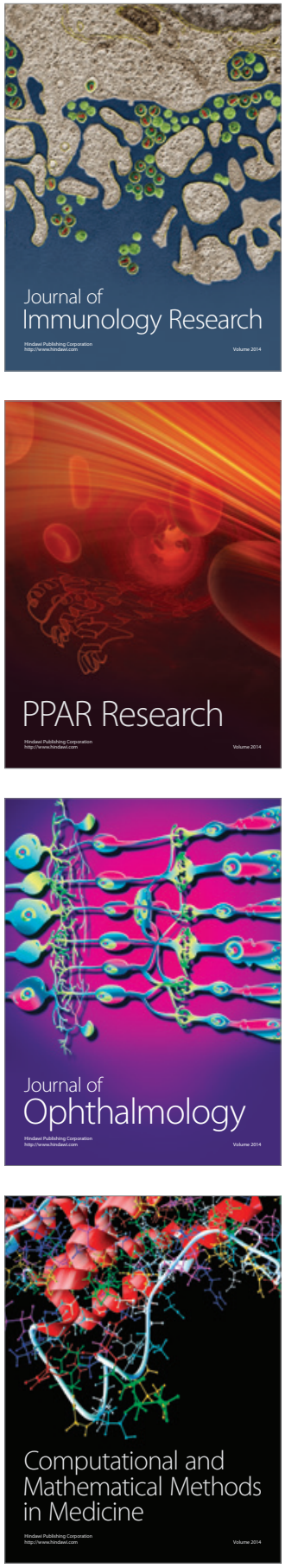

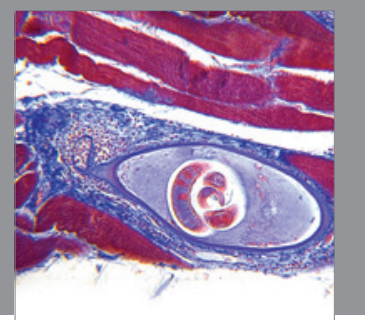

Gastroenterology

Research and Practice
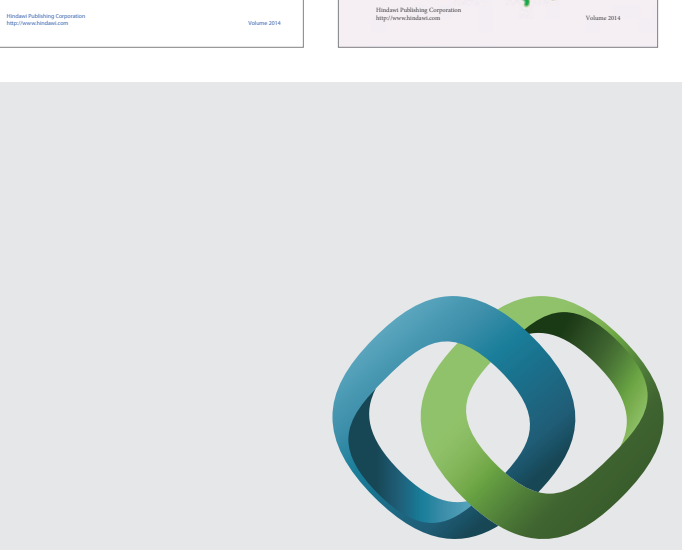

\section{Hindawi}

Submit your manuscripts at

http://www.hindawi.com
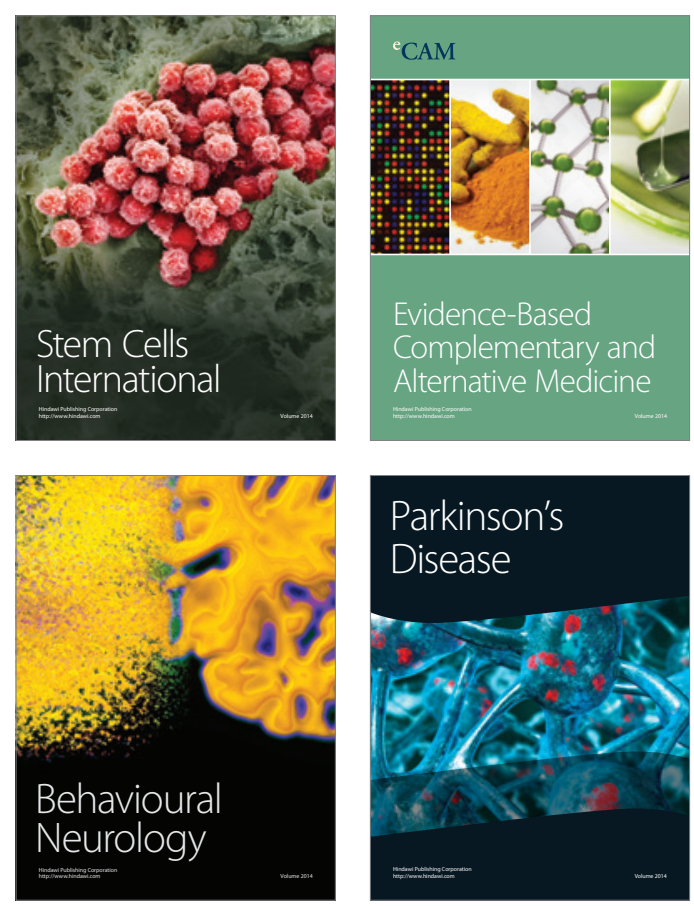

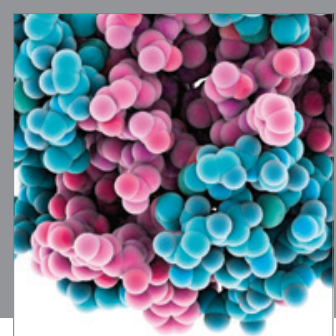

Journal of
Diabetes Research

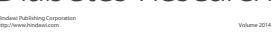

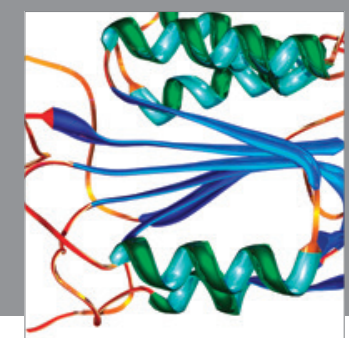

Disease Markers
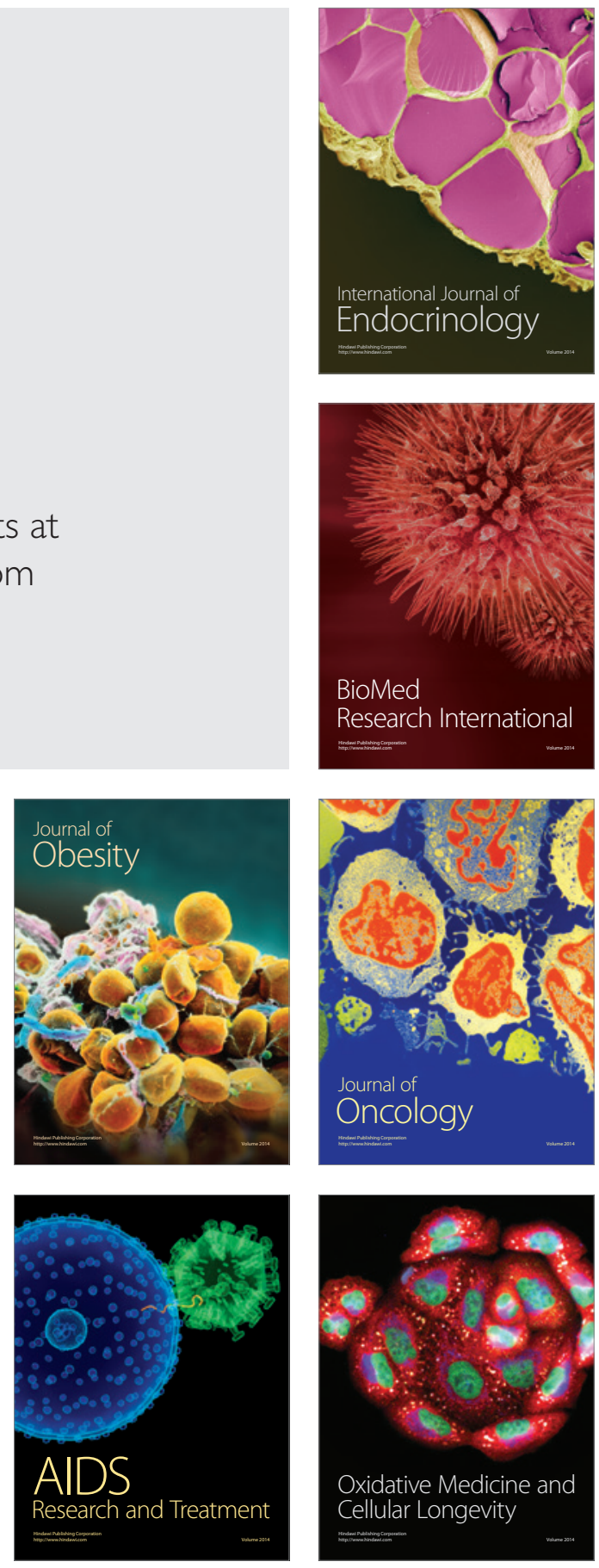